\title{
Analog MIMO Radio-Over-Copper Downlink With Space-Frequency to Space-Frequency Multiplexing for Multi-User 5G Indoor Deployments
}

\author{
Andrea Matera and Umberto Spagnolini, Senior Member, IEEE
}

\begin{abstract}
Radio access network (RAN) centralization is at the basis of current mobile networks, in which BaseBand Units (BBUs) and radio antenna units (RAUs) exchange over the FrontHaul (FH) digitized radio-frequency signals through protocols such as the common public radio interface. However, such architecture, as it stands, does not scale to the demands of multiple-antennas 5G systems, thus leading to drastic RAN paradigm changes. Differently from digital RAN architectures, we propose to overcome bandwidth/latency issues due to digitization by employing an all-analog FH for multiple-antenna RAUs based on the analog radio-over-copper (A-RoC) paradigm. The A-RoC is an alternative/complementary solution to $\mathrm{FH}$ for the last $200 \mathrm{~m}$, such as for indoor, to reuse existing local area network (LAN) cables with remarkable economic benefits. Although LAN cables contain 4 twisted-pairs with up to $500 \mathrm{MHz}$ bandwidth/ea., their usage is limited by cable attenuation and crosstalk among pairs. This paper demonstrates that a judicious mapping of each radio-frequency signal of each antenna onto a combination of cable pairfrequency allocations, referred to as space-frequency to spacefrequency multiplexing, optimized together with the design of the digital precoding at the $\mathrm{BBU}$, substantially mitigates the cable impairments. The LAN cables can be exploited for last 100-200 $\mathrm{m}$ analog transport $\mathrm{FH}$ to meet the requirements of $5 \mathrm{G}$ indoor networks.
\end{abstract}

Index Terms-Radio-over-copper, MIMO, C-RAN, resource allocation, analog fronthauling, indoor coverage.

\section{INTRODUCTION}

$\mathbf{N}$ EXT Generation (5G and beyond) wireless communications systems will be designed to smartly connect "anyone, any device, anywhere", incorporating any other 3 GPP (i.e., LTE) and non-3GPP (i.e., WiFi) technologies to provide extensive high-rate connectivity and a seamless user experience to all the interconnected user devices. According to [1], $96 \%$ of $5 \mathrm{G}$ wireless traffic will originate or terminate within a building, and this is the reason why providing bandwidth- and cost-efficient solutions for enhanced indoor coverage has a key role in developing next generation wireless networks.

Centralized Radio Access Network (C-RAN) is an attractive solution to handle complex interference scenarios through the

Manuscript received September 17, 2018; revised January 21, 2019; accepted March 24, 2019. Date of publication April 9, 2019; date of current version May 8, 2019. The associate editor coordinating the review of this paper and approving it for publication was M. S. Alouini. (Corresponding author: Andrea Matera.)

The authors are with the Dipartimento di Elettronica, Informazione e Bioingegneria, Politecnico di Milano, 20133 Milan, Italy (e-mail: andrea.matera@polimi.it; umberto.spagnolini@polimi.it). colocation of BaseBand Units (BBUs) and the remotization of antennas in so-called Remote Antenna Units (RAUs), densely geographically distributed both indoor and outdoor, thus allowing for centralized signal processing, network scalability, and costs reduction [2]. BBU and RAU exchange over the FrontHaul (FH) in-phase and quadrature RadioFrequency (RF) signals, after these are digitized according to any of the routinely employed protocols, such as the Common Public Radio Interface (CPRI) [3]. However, the expected increase in RF signal bandwidth and the massive number of antennas call into question the effectiveness of such digital FH architecture, which cannot be simply tailored to meet the bandwidth, synchronization, and latency requirements of 5G mobile FH [4], [5].

A promising direction is to redistribute the RAN functionalities between BBU and RAU, trading over bandwidth/latency requirements, advanced Cooperative Multi-Point (CoMP) processing capability, and RAU cost/complexity [6]. In particular, intra-PHYsical layer (PHY) functional split options shift some PHY functions from BBU to RAU, e.g., resource mapping, Fourier transform, etc. These enable CoMP techniques among multiple RAUs with relaxed bandwidth constraints, while ensuring the same low complexity at the RAUs [7], [8]. Intra-PHY functional splits broaden the number of possible options, and loose some interoperability benefits of plain full-digital CPRI. The CPRI forum itself is currently working on the definition of the enhanced CPRI (eCPRI) transport FH interface based on intra-PHY splits [9]. However, the conclusion is that there is no one-solution-fits-all for 5G RAN, but it rather needs to be specifically designed to comply with bandwidth, latency, and budget required by each specific scenario [5].

In contrast with the newly proposed $\mathrm{FH}$ architectures, which are mostly digital, one viable solution for high-rate/lowlatency/synchronized RAU applications is to employ a fully analog FH link [10]-[12]. Focusing on the uplink, in ana$\log \mathrm{FH}$, the RAUs first down-covert the RF signals received from the User Equipments (UEs) to a lower frequency, known as Intermediate Frequency (IF), and then relay these IF signals to the BBU for baseband processing. The downlink is symmetrical: the RAUs receive the IF signals from the BBU, perform up-conversion, and relay the RF signals to the UEs. All-analog processing avoids any bandwidth expansion due to digitization or latency (up to the propagation only), enables fairly precise bit/carrier-frequency synchronized RAUs, 
simplifies the FH, reduces hardware costs, and improves energy efficiency.

Analog Radio-over-Fiber (A-RoF) is a promising example of analog FH, due to its capability to carry several Gbit/s in terms of equivalent data-rate [11], [13]. However, the deployment of a large-scale and pervasive optic infrastructure is too costly to provide a satisfactory business case for indoors, pushing to look for novel cost-effective solutions such as Analog Radio-over-Copper (A-RoC), which has been gaining much attention over the last years [10], [14], [15], and it is the focus of this paper.

\section{A. Related Works and Motivation}

A-RoC has been proposed in Gambini and Spagnolini [12], where existing twisted-pairs copper cables were considered for indoor femto-cell systems. In [12], analog RF signals are exchanged between a remote location hosting all the PHY/Medium Access Control (MAC) functionalities (i.e., BBU) and an in-home device performing only the analog relay of signals to antennas (i.e., RAU).

Afterwards, A-RoC gained lots of attention becoming the basis of indoor commercial solutions [14] which exploit the Local Area Network (LAN) cables already deployed in buildings and allow to extend the indoor coverage over distances longer than $100 \mathrm{~m}$. By utilizing all the 4 twisted-pairs bonded together into the cable at low-frequency (characterized by low attention and crosstalk interference), one can serve up to 4 antennas (e.g., $4 \times 4 \mathrm{MIMO}$ ) per LAN cable.

Still based on the A-RoC concept, Huang et al. [16] proposed an LTE-over-copper architecture based on the colocation of RAU and Digital Subscriber Line (DSL) equipment in the same street cabinets. Authors proved that, by employing a single-twisted pair in the $21-24 \mathrm{MHz}$ cable frequency band (not used by any DSL service), it is feasible to transport a 3GPP compliant LTE radio signal up to $350 \mathrm{~m}$ away from the cabinet. Crosstalk mitigation in LTE-over-copper systems is covered in Medeiros et al. [17] for the case of 6 twisted-pairs interfering each other, still in the $21-24 \mathrm{MHz}$ low-frequency range of cable.

Even if the aforementioned works demonstrated the feasibility of A-RoC for the FH, none of them attempted to push the usage of cable frequency beyond the very first few tens of $\mathrm{MHz}$, thus not fully exploiting the bandwidth capabilities of copper cables. The high frequency portion of LAN cables (i.e., Cat-5/6/7) for C-RAN applications has been first explored in Naqvi et al. [18], showing by realistic numerical simulations that up to $60 \mathrm{RAU}$ antennas carrying a $20 \mathrm{-MHz}$ LTE channel/ea. can be served by a $100 \mathrm{~m}$ Cat -5 cable with approx. $500 \mathrm{MHz}$ bandwidth/pair. Recently, a prototype has been developed to demonstrate experimentally the feasibility of relaying in an all-analog fashion multiple MIMO signals over LAN cables up to $400 \mathrm{MHz}$ cable frequency [19], thus validating the conceptual architecture proposed in [18].

The analog C-RAN paradigm presented in [18] and [19], referred to as A-MIMO-RoC due to the cascade of a MIMO cable channel over a MIMO wireless channel, encompasses both the advantages of analog $\mathrm{FH}$ and the cost benefits of
LAN cables [15]. However, in practice, the design and optimization of A-MIMO-RoC poses several technical challenges: i) crosstalk interference arising among the 4 twisted-pairs at the opposite ends of the cable binder, denoted as Far-End CrossTalk (FEXT), and cable attenuation, denoted as Insertion Loss (IL), severely limit the performance of LAN cables, if not properly handled [18], ii) for a large number of RAU antennas, the mapping of the signal to/from each antenna onto the available cable resources becomes a major problem to be solved, iii) the RAU equipment (e.g., home-device) should be as simple/cheap as possible, but in the meantime able to handle up to several tens of antennas, $i v$ ) LAN cables are subject to strict power constraints that must be carefully taken care of in the system design, especially in downlink direction, and v) in case of multiple-users, interference cancellation techniques for compound A-MIMO-RoC should be properly designed, but still relieving the RAU from any computationally complex signal processing.

In previous works [20], [21], there is a single-user uplink A-MIMO-RoC architecture based on LAN cables to address the critical aspects $i$ ), ii) and iii) mentioned above. In particular, [20] demonstrated that by optimally mapping the RF signals of each RAU antenna onto a combination of twisted-pair/frequency allocations over the cable, referred to as Space-Frequency to Space-Frequency (SF2SF) multiplexing, it is possible to substantially mitigate the impairments introduced by the cable $\mathrm{FH}$, once evaluated in terms of enduser throughput. In [21], the coexistence over the same LAN cable between FH signals and other services (e.g., DSL, PoE, etc.) has been tested for indoor, thus confirming the performance boost provided by SF2SF multiplexing. Finally, still focusing on the A-MIMO-RoC uplink, [22] presents an information-theoretical study for heterogeneous $5 \mathrm{G}$ networks characterized by different traffic types and constraints. In particular, [22] provides some useful insights on the performance trade-offs between enhanced Mobile BroadBand (eMBB) and Ultra Reliable Low Latency Communications (URLLC) services, when they coexist in the uplink of the proposed A-MIMO-RoC architecture.

Beyond A-MIMO-RoC with SF2SF for uplink FH scenarios, there are several open issues of practical interest, such as the A-MIMO-RoC downlink system design and the multiuser setting (complicated by power constraints over cables). Preliminary results on the A-MIMO-RoC downlink for a simple single-user scenario are in [23], but the downlink A-MIMO-RoC problem for multi-user configuration poses issues that have never been considered before, and these are the focus of this paper. In multi-user settings, the downlink problem requires an overall system optimization over the cascade of two MIMO channels (i.e., the FH cable channel and the wireless channel), and problem resembles the optimization of a two-hop Amplify-and-Forward (AF) MIMO relay system, first addressed in [24] and [25]. Among the numerous valuable works dealing with AF systems that followed [24], [25] (for an excellent survey on the topic we refer the reader to [26] and references herein), the one by Chae et al. [27] is of particular interest for our purposes, since it focuses on the downlink channel of a cellular system in which the base station 
communicates with multiple single-antenna users through a single-relay node equipped with multiple-transceivers.

This paper investigates the downlink multi-user A-MIMORoC architecture for $5 \mathrm{G}$ indoor deployments by optimizing the AF structure that is constrained to perform the mapping of radio carriers for each antenna onto suitably chosen IL/FEXT impaired resources over the copper FH.

\section{B. Contributions}

The contributions of this paper are three-fold: $i)$ we propose a downlink A-MIMO-RoC architecture compliant with standardized LAN cables and heterodyne-only low-complexity RAU for multi-user settings; $i$ ) we propose a system design based on the optimization of the SF2SF multiplexing between air- and cable-link resources combined with the BBU precoder design, and iii) we validate numerically the proposed architecture on geometrical air-link MIMO channel models and $100 \mathrm{~m}$ cable (as typical reference length in copper cable community); performance are compared with the Singular Value Decomposition (SVD) relay design scheme proposed in [27], assumed here as a performance benchmark. In particular, we remark the differences with respect to our previous work [23], which presents a single-user A-MIMO-RoC downlink architecture, while this paper focuses on multi-user downlink settings. Notice that the extension of [23] to multiuser settings is far from trivial as: $i$ ) the SF2SF multiplexing problem needs to be combined with the design of the precoding algorithm for multi-user interference cancellation (see Sect. III); ii) as a consequence, transmit power constraints must be fulfilled not only at the RAU antennas (as in [23]), but jointly at the cable input at the BBU (see Sect. II-D), iii) the SF2SF optimization problem needs to be extended to account for different performance metrics (e.g., sum-rate, minimum user rate, etc.) and for different precoding algorithms (see Sect. IV-B).

\section{Organization}

Sect. II describes the A-MIMO-RoC downlink system model, detailing both the radio channel and the spacefrequency copper-based fronthauling, and introducing the idea of SF2SF multiplexing. The BBU precoding techniques are in Sect. III, while Sect. IV discusses the proposed SF2SF multiplexing optimization. Simulation results are in Sect. V. Sect. VI concludes the paper.

\section{Notation}

Bold upper- and lower-case letters describe matrices and column vectors. The $i j$-th element of matrix $\mathbf{A}$ is denoted as $[\mathbf{A}]_{i j}$. The $i$-th element of vector $\mathbf{a}$ is denoted as $a_{i}$ or, if confusion may arise, as $[\mathbf{a}]_{i}$. Letters $\mathbb{R}$ and $\mathbb{C}$ refer to real and complex numbers, respectively. We denote matrix inversion, transposition and conjugate transposition as $(\cdot)^{-1},(\cdot)^{T},(\cdot)^{H}$. Matrix $\mathbf{I}$ is an identity matrix of appropriate size and $\mathrm{E}[\cdot]$ is the statistical expectation. Notation $\operatorname{diag}(\mathbf{A})$ denotes a diagonal matrix containing the main diagonal elements of matrix $\mathbf{A}, \operatorname{diag}\left(\mathbf{A}_{1}, \mathbf{A}_{2}, \ldots, \mathbf{A}_{N}\right)$ is a block-diagonal

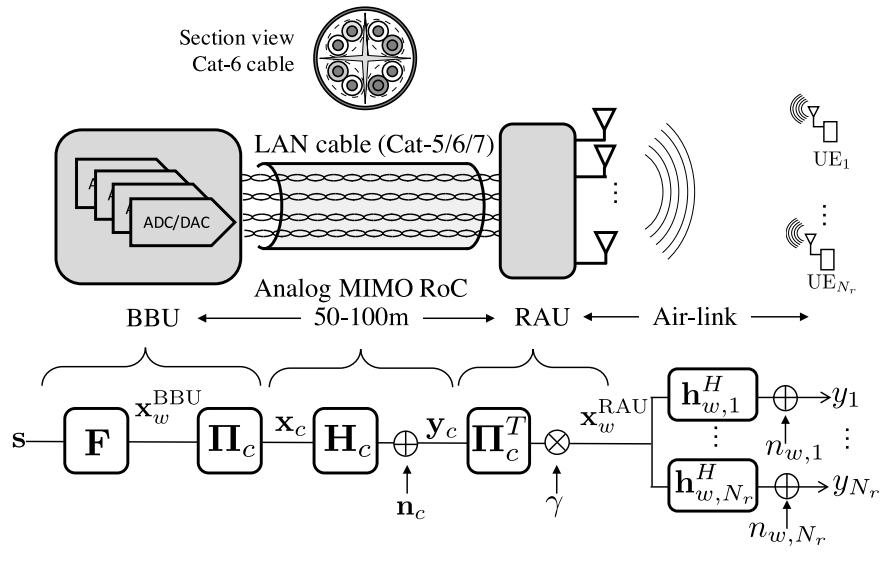

Fig. 1. System model: multi-user downlink A-MIMO-RoC architecture based on LAN cables.

matrix with the main diagonal blocks given by matrices $\mathbf{A}_{1}, \mathbf{A}_{2}, \ldots \mathbf{A}_{N}$, and $\operatorname{diag}\left(a_{1}, a_{2}, \ldots, a_{N}\right)$ is a diagonal matrix with elements $a_{1}, a_{2}, \ldots, a_{N}$ on the main diagonal. Finally, $\|\mathbf{a}\|_{2}=\sqrt{\sum_{i}\left|a_{i}\right|^{2}}$ is the $l_{2}$ - norm of vector $\mathbf{a}$.

\section{SySTEM MODEL}

The A-MIMO-RoC fronthauling for C-RAN architecture is presented in Fig. 1. The system is a cascade of cable and wireless links, denoted by subscript $(\cdot)_{c}$ and $(\cdot)_{w}$, respectively. Here we focus on the downlink, where the BBU communicates with $N_{r}$ UEs through a RAU equipped with $N \geq N_{r}$ antennas, which acts as an AF relay node comprehensive of frequency conversions. Without any loss of generality, UEs are singleantenna (or single-stream).

The downlink signals are precoded at the BBU, before being channelized into Space and Frequency Division Multiplexing (SDM and FDM, respectively) over the 4-pair wired access link. These are transmitted over the air-link by the RAU. Similarly to [18], we assume that each of the $N$ RAU antennas carries one wireless signal with bandwidth $B_{w}$ so that, overall, at least

$$
B_{c} \geq N \cdot B_{w}
$$

cable bandwidth is needed to transport all the $\mathrm{FH}$ radio signal. The inequality in (1) provides degrees of freedom to cope with cable impairments (Sect. III). The system in Fig. 1 can thus be regarded as a chain of two heterogeneous channels with different peculiarities.

\section{A. Radio Channel}

For reasoning simplicity of this section, let the downlink radio channel RAU $\rightarrow$ UEs be narrow-band frequencyflat block-fading (e.g., it might correspond to one sub-carrier of OFDM modulations) such that the signal received at the $k$-th UE

$$
y_{k}=\mathbf{h}_{w, k}^{H} \mathbf{x}_{w}^{\mathrm{RAU}}+n_{w, k}
$$

involves the channel vector for the $k$-th $\mathrm{UE} \mathbf{h}_{w, k} \in \mathbb{C}^{N \times 1}$, the additive white Gaussian noise $n_{w, k} \sim \mathcal{C N}\left(0, \sigma_{w}^{2}\right)$, and the 
signal transmitted by the RAU $\mathbf{x}_{w}^{\mathrm{RAU}} \in \mathbb{C}^{N \times 1}$ subject to the average (wireless) power constraint

$$
\mathrm{E}\left[\left\|\mathbf{x}_{w}^{\mathrm{RAU}}\right\|_{2}^{2}\right] \leq P_{w} .
$$

In this paper, we consider a geometrical channel model based on the Angles of Departure (AoDs) of signals [28], which accurately captures the mathematical structure of a mm-wave systems (say $>6-10 \mathrm{GHz}$ ) but it can also be extended to lower spectrum systems. In particular, it is assumed here that the channel $\mathbf{h}_{w, k}$ from the RAU to the $k$-th $\mathrm{UE}$ is the sum of the contribution of $L_{k}$ propagating paths as

$$
\mathbf{h}_{w, k}=\rho_{k} \sum_{l=1}^{L_{k}} \alpha_{k, l} \mathbf{a}\left(\phi_{k, l}, \theta_{k, l}\right),
$$

where $\rho_{k}=\sqrt{N / L_{k}}$ is a normalization factor, $\alpha_{k, l} \sim$ $\mathcal{C N}(0,1)$ is the complex gain for the $l$-th path, and $\mathbf{a}\left(\phi_{k, l}, \theta_{k, l}\right)$ is the array response toward the $k$-th UE with $\phi_{k, l}$ and $\theta_{k, l}$ denoting the azimuth and elevation AoDs, respectively. Considering half-wavelength spaced antenna elements, the array response of the $D_{1} \times D_{2}$ Uniform Planar Array (UPA) is the $N \times 1$ vector

$$
\begin{aligned}
\mathbf{a}_{\mathrm{UPA}}(\phi, \theta)= & \frac{1}{\sqrt{N}}\left[1, \ldots, e^{j \pi\left(d_{1} \sin \phi \sin \theta+d_{2} \cos \theta\right)}, \ldots,\right. \\
& \left.e^{j \pi\left(\left(D_{1}-1\right) \sin \phi \sin \theta+\left(D_{2}-1\right) \cos \theta\right)}\right]^{T},
\end{aligned}
$$

with $d_{1} \in\left\{0,1, \ldots, D_{1}-1\right\}, d_{2} \in\left\{0,1, \ldots, D_{2}-1\right\}$ and $N=D_{1} D_{2}$.

\section{B. Space-Frequency Cable Fronthaul Channel}

The LAN cable CAT models are known in cable community, and these are very accurate in describing both FEXT and IL with minimal variability [29]. The same model has been adopted here for downlink FH cable channel by re-adapting notation and setup to the problem at hand. The adaptation, originally presented in our previous work [23], is detailed in this section. Targeting no digital-processing at the RAU implies that its functionalities are limited to signal amplification, with possibly intra-cable frequency bands swapping to comply with cable capability (Sect. IV).

The transmit signal $\mathbf{x}_{w}^{\mathrm{RAU}}$ is just a scaled and frequency upconverted version of the signal

$$
\mathbf{y}_{c}=\mathbf{H}_{c} \mathbf{x}_{c}+\mathbf{n}_{c},
$$

received from the BBU over the $\mathrm{FH}$ wired access link $\mathbf{H}_{c} \in$ $\mathbb{C}^{N_{c} \times N_{c}}$, where $\mathbf{n}_{c} \sim \mathcal{C N}\left(\mathbf{0}, \sigma_{c}^{2} \mathbf{I}\right)$ is the uncorrelated white Gaussian cable noise with power $\sigma_{c}^{2}$ and $\mathbf{x}_{c} \in \mathbb{C}^{N_{c} \times 1}$ is the signal transmitted by the BBU over the cable.

The FH cable channel $\mathbf{H}_{c}$ is equivalent to a large MIMO system in the Space-Frequency (SF) domain defined by $N_{s}$ SDM channels (i.e., the $N_{s}$ twisted-pairs bonded together into the cable, $N_{s}=4$ is typical for Cat -6 LAN cables) and $N_{f}$ FDM channels for a total of $N_{c}=N_{s} \cdot N_{f}$ SF resource blocks on cable. To simplify the discussion here, it is assumed that the total number of available SF cable channels $N_{c}$ exactly equals the total number of RAU antennas $N$ (i.e., $N_{c}=N$ ), even if in practice it could be relaxed to $N_{c}>N$ with further

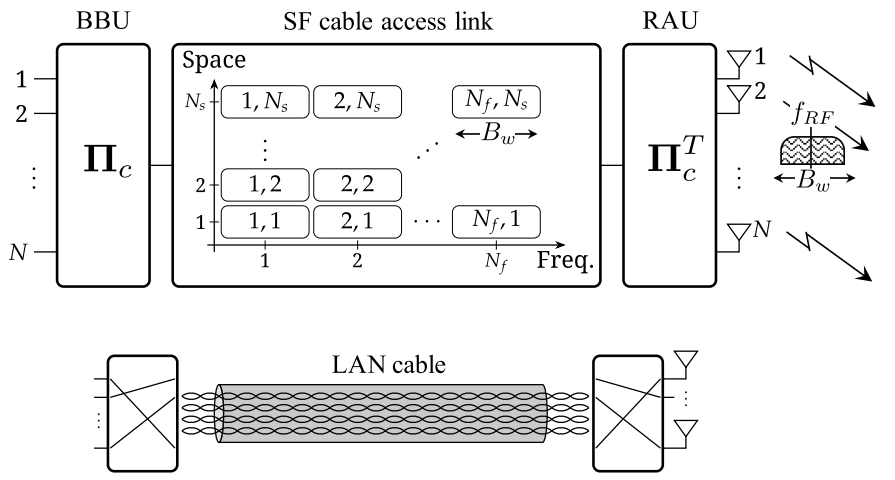

Fig. 2. Space-Frequency wired access link: (upper) mathematical and (lower) physical model.

benefits. The matrix cable channel $\mathbf{H}_{c}$ accounts for the cable IL (diagonal terms, $\left[\mathbf{H}_{c}\right]_{i, i}$ ) and for the cable FEXT among all the $N$ cable channels (off-diagonal terms, $\left[\mathbf{H}_{c}\right]_{i, j}$ ). Since cross-cable interference occurs only among spatial channels at the same frequency (i.e., frequency bands are disjointed), it is straightforward to describe $\mathbf{H}_{c}$ by the block diagonal matrix

$$
\mathbf{H}_{c}=\operatorname{diag}\left(\mathbf{H}_{c, 1}, \mathbf{H}_{c, 2}, \ldots, \mathbf{H}_{c, N_{f}}\right),
$$

where each of the $N_{f}$ diagonal blocks $\mathbf{H}_{c, f} \in \mathbb{C}^{N_{s} \times N_{s}}$ is the cable channel matrix at the $f$-th frequency band. In particular, the main diagonal element $\left[\mathbf{H}_{c, f}\right]_{i i}$ contains the IL for the $i$-th twisted-pair at the $f$-th frequency band, while the offdiagonal element $\left[\mathbf{H}_{c, f}\right]_{i j}$, with $i \neq j$, denotes the FEXT between twisted-pair $j$-th and $i$-th at $f$-th frequency band.

The BBU signal $\mathbf{x}_{c}$ is transmitted to the RAU over the SF cable resource to be allocated, hence, it is conveniently defined as

$$
\mathbf{x}_{c}=\left[\mathbf{x}_{c, 1}^{T}, \mathbf{x}_{c, 2}^{T}, \ldots, \mathbf{x}_{c, N_{f}}^{T}\right]^{T},
$$

where $\mathbf{x}_{c, f} \in \mathbb{C}^{N_{s} \times 1}$ are the symbols transmitted over the $N_{s}$ twisted-pairs at the $f$-th frequency band. In particular, $\left[\mathbf{x}_{c, f}\right]_{j}$ denotes the BBU symbol transmitted over the $j$-th twisted-pair at the $f$-th cable frequency band. The cable symbols $\mathbf{x}_{c}$ are obtained at the BBU by precoding $(\mathbf{F})$ and mapping $\left(\boldsymbol{\Pi}_{c}\right)$ onto the $N$ SF cable channels the $N_{r}$ uncorrelated and normalized to unit-power UE information symbols $\mathbf{s}=\left[s_{1}, s_{2}, \ldots, s_{N_{r}}\right]^{T}$ as

$$
\mathbf{x}_{c}=\boldsymbol{\Pi}_{c} \mathbf{x}_{w}^{\mathrm{BBU}}, \quad \mathbf{x}_{w}^{\mathrm{BBU}}=\mathbf{F} \mathbf{s} .
$$

Permutation matrix $\boldsymbol{\Pi}_{c}$ maps the precoded symbols $\mathbf{x}_{w}^{\mathrm{BBU}}$ intended to the $N$ RAU antennas onto $N$ SF blocks over the cable properly selected according to SF2SF multiplexing as in Sect. III. In other words, $\left[\mathbf{x}_{w}^{\mathrm{BBU}}\right]_{n}$ denotes the BBU symbol that is relayed over the cable towards the $n$-th RAU antenna. Recall that each of the $N$ RAU antennas carries a signal with bandwidth $B_{w}$. Eq. (9) thus describes how the radio signal $\mathbf{s}$ is transported from the BBU to the $N$ RAU antennas after being mapped over the $N$ SF cable FH channels. To comply with standardized cable constraints [30], $\mathbf{x}_{c}$ is subject to Per-Line Per-Carrier (PLPC) transmit power constraints at the cable input, also known as Power Spectral Density (PSD) masks, 
as

$$
\mathrm{E}\left[\left|\left[\mathbf{x}_{c, f}\right]_{j}\right|^{2}\right] \leq P_{c}, \quad \forall f=1, \ldots, N_{f}, \forall j=1, \ldots, N_{s},
$$

and also per-line Aggregate Transmit Power (ATP) constraints

$$
\sum_{f=1}^{N f} \mathrm{E}\left[\left|\left[\mathbf{x}_{c, f}\right]_{j}\right|^{2}\right] \leq P_{A T P}, \quad, \forall j=1, \ldots, N_{s} .
$$

Both the technological constraints typical of copper cables [30] complicate the SF2SF design.

Permutations $\boldsymbol{\Pi}_{c}$ are mutually signaled between RAU and BBU: once the specific SF2SF multiplexing $\Pi_{c}$ is chosen at the BBU, this information is signaled to the RAU that scales the signal power to match the power constraints and maps back the symbols from the SF cable blocks to the corresponding antennas by performing an inverse permutation $\mathbf{x}_{w}^{\mathrm{RAU}}=\gamma \boldsymbol{\Pi}_{c}^{T} \mathbf{y}_{c}$. Hence, the overall model describing the communication between RAU and BBU over cable is described by

$$
\mathbf{x}_{w}^{\mathrm{RAU}}=\gamma \mathbf{H}_{S F} \mathbf{x}_{w}^{\mathrm{BBU}}+\gamma \mathbf{n}_{c},
$$

where $\mathbf{H}_{S F}=\boldsymbol{\Pi}_{c}^{T} \mathbf{H}_{c} \boldsymbol{\Pi}_{c}$ is the equivalent $\mathrm{SF}$ permuted cable channel, statistical properties of $\mathbf{n}_{c}$ are invariant for permutations so that $\boldsymbol{\Pi}_{c}^{T} \mathbf{n}_{c} \rightarrow \mathbf{n}_{c}$, and $\gamma$ is the power scaling factor.

\section{Space-Frequency to Space-Frequency Multiplexing}

This subsection first reviews the mathematical model introduced in [23] for the $\mathrm{FH}$ resources allocation, and then provides a simple working example just to gain insights. In the considered A-MIMO-RoC architecture, we assume that the only feasible degree of freedom at the low-cost/complexity/latency RAU in mitigating the cable FH impairments is the resource allocation between the SF resources over the cable and the SF resources over the wireless link, referred here to as SF2SF multiplexing. This multiplexing operation origins at the $\mathrm{BBU}$ and determines on which cable SF channel each of the precoded signals $\mathbf{x}_{w}^{\mathrm{BBU}}$ must be relayed efficiently to the RAU. This is described by

$$
\mathbf{x}_{c}=\boldsymbol{\Pi}_{c} \mathbf{x}_{w}^{\mathrm{BBU}},
$$

where the permutation $\boldsymbol{\Pi}_{c} \in\{0,1\}^{N \times N}$ is a unitary (i.e., $\left.\boldsymbol{\Pi}_{c} \boldsymbol{\Pi}_{c}^{T}=\boldsymbol{\Pi}_{c}^{T} \boldsymbol{\Pi}_{c}=\mathbf{I}\right)$ binary matrix having a single non-zero entry per row/column and describing how the BBU symbols $\mathbf{x}_{w}^{\mathrm{BBU}}$, to be later transmitted by the $N$ RAU antennas, are mapped onto the cable transmit symbols $\mathbf{x}_{c}$ in both SDM and FDM. Consistently with the definition of the cable symbols $\mathbf{x}_{c}$, the following notation is adopted to highlight the partitioning in mapping operation performed by $\boldsymbol{\Pi}_{c}$ :

$$
\begin{aligned}
\boldsymbol{\Pi}_{c}=[\underbrace{\mathbf{p}_{11}, \ldots, \mathbf{p}_{1 N_{s}}}_{1^{\text {st }} \text { freq }}, \ldots, \underbrace{\mathbf{p}_{f 1}, \ldots, \mathbf{p}_{f N_{s}}}_{f^{\text {th }} \text { freq }}, \ldots, \\
\underbrace{\mathbf{p}_{N_{f} 1}, \ldots, \mathbf{p}_{N_{f} N_{s}}}_{N_{f}^{\text {th }} \text { freq }}]^{T} .
\end{aligned}
$$

The row of $\boldsymbol{\Pi}_{c}$ is $\mathbf{p}_{f j}^{T}=\left[p_{f j}^{1}, p_{f j}^{2}, \ldots, p_{f j}^{N}\right]$, where the single non-zero entry $p_{f j}^{n}$ selects the BBU symbol $\left[\mathbf{x}_{w}^{\mathrm{BBU}}\right]_{n}$ to be relayed toward the UEs by the $n$-th RAU antenna after being transmitted to the RAU over the $j$-th pair at the $f$-th frequency band of the copper cable:

$$
\left[\mathbf{x}_{c, f}\right]_{j}=\mathbf{p}_{f j}^{T} \mathbf{x}_{w}^{\mathrm{BBU}} .
$$

Each permutation matrix $\boldsymbol{\Pi}_{c}$ is univocally identified by a vector $\pi=\left[\pi_{1}, \pi_{2}, \ldots, \pi_{N}\right]^{T}$ storing all the indexes of the non-zero elements of the rows of matrix $\boldsymbol{\Pi}_{c}$, i.e., $\pi_{n}$ is the index of the non-zero element of the $n$-th row of matrix $\boldsymbol{\Pi}_{c}$.

Example: To gain some insights on the multiplexing performed by $\boldsymbol{\Pi}_{c}$, let us assume a very simple $4 \times 4$ reference channel model $\mathbf{H}_{c}$ compound of 2 frequencies defined as

$$
\mathbf{H}_{c}=\left[\begin{array}{cccc}
\alpha_{(1,1)} & \beta_{1} & 0 & 0 \\
\beta_{1} & \alpha_{(2,1)} & 0 & 0 \\
0 & 0 & \alpha_{(1,2)} & \beta_{2} \\
0 & 0 & \beta_{2} & \alpha_{(2,2)}
\end{array}\right],
$$

where $\alpha_{(i, f)}=\left[\mathbf{H}_{c, f}\right]_{i i}$ is the IL coefficient for the $i$ th twisted-pair $\left(\operatorname{tp}_{i}\right)$ at the $f$-th cable frequency band $\left(\mathrm{f}_{f}\right)$ and $\beta_{f}=\left[\mathbf{H}_{c, f}\right]_{i j}, \forall i \neq j$ is the FEXT coefficient at $\mathbf{f}_{f}$ (for simplicity in this example we assume symmetric FEXT coefficients at each frequency, although this is not true in general). Let us consider a BBU signal $\mathbf{x}_{w}^{\mathrm{BBU}} \in \mathbb{C}^{4 \times 1}$ which is first transmitted over the cable and then relayed to the UEs by a RAU equipped with 4 antennas, and assume the following desired SF2SF multiplexing between cable and air resources: $\left(\mathrm{tp}_{1}, \mathrm{f}_{2}\right) \rightarrow \mathrm{Ant}_{1},\left(\mathrm{tp}_{1}, \mathrm{f}_{1}\right) \rightarrow \mathrm{Ant}_{2},\left(\mathrm{tp}_{2}, \mathrm{f}_{2}\right) \rightarrow \mathrm{Ant}_{3}$, $\left(\mathrm{tp}_{2}, \mathrm{f}_{1}\right) \rightarrow \mathrm{Ant}_{4}$, where $\left(\mathrm{tp}_{i}, \mathrm{f}_{f}\right) \rightarrow \mathrm{Ant}_{n}$ means that the BBU signal $\left[\mathbf{x}_{w}^{\mathrm{BBU}}\right]_{n}$, that has to be relayed to the UEs by the $n$ th RAU antenna $\mathrm{Ant}_{n}$, is transmitted to the RAU over the $i$-th cable pair at the $f$-th frequency band. This operation is performed by the permutation

$$
\boldsymbol{\Pi}_{c}=\left[\begin{array}{llll}
0 & 1 & 0 & 0 \\
0 & 0 & 0 & 1 \\
1 & 0 & 0 & 0 \\
0 & 0 & 1 & 0
\end{array}\right]
$$

leading to the permuted channel matrix $\mathbf{H}_{S F}=\boldsymbol{\Pi}_{c}^{T} \mathbf{H}_{c} \boldsymbol{\Pi}_{c}$. Hence, according to (12), the transmitted signal at the RAU yields

$$
\overbrace{\left[\begin{array}{l}
x_{w, 1} \\
x_{w, 2} \\
x_{w, 3} \\
x_{w, 4}
\end{array}\right]}^{\mathbf{x}_{w}^{\mathrm{RAU}}}=\gamma \cdot\left[\begin{array}{cccc}
\alpha_{(1,2)} & 0 & \beta_{2} & 0 \\
0 & \alpha_{(1,1)} & 0 & \beta_{1} \\
\beta_{2} & 0 & \alpha_{(2,2)} & 0 \\
0 & \beta_{1} & 0 & \alpha_{(2,1)}
\end{array}\right] \overbrace{\left[\begin{array}{l}
x_{w, 1} \\
x_{w, 2} \\
x_{w, 3} \\
x_{w, 4}
\end{array}\right]}^{\mathbf{x}_{w}^{\mathrm{BBU}}}+\mathbf{n}_{c},
$$

which shows that the above mapping rules are met: e.g., the BBU signal $\left[\mathbf{x}_{w}^{\mathrm{BBU}}\right]_{2}$ is relayed over the $1^{\text {st }}$ pair at the $1^{\text {st }}$ frequency band and then transmitted by the $2^{\text {nd }}$ RAU antenna, $\left(\mathrm{tp}_{1}, \mathrm{f}_{1}\right) \rightarrow \mathrm{Ant}_{2}$. However, it is important to notice also that the relay over the cable introduces interference among the BBU symbols which needs to be carefully handled. The aim of this work is to show that by a proper SF2SF multiplexing, to be optimized in combination with the design of the precoder $\mathbf{F}$, 
this interference can be mitigated, leading to significant performance improvement at virtually no-cost at RAU, and transparently to the end-to-end link.

\section{Power Constraints}

Differently from previous works (e.g., [23]), here cable and wireless power constraints are jointly fulfilled for multi-user hybrid wireless-wired MIMO AF system. More specifically, these are: $i$ ) a total power constraint at the RAU (3), ii) PLPC power constraints at the input of the cable channel (10) and iii) ATP constraint over each twisted-pair (11). Consistently with the assumption of unit mean power constellation symbols, we consider here a total power constraint at the RAU $P_{w}=N$ (i.e., unit mean power per transmitting antenna) as well as unit power PLPC constraints $P_{c}=1$ at the cable input. Notice that, differently from the multi-user settings considered here, for the single-user case the constraints $i i)$ do not represent an obstacle, as they are always fulfilled by the choice of the beamforming vector [23].

PSD optimization is beyond the scope of this paper (we refer interest readers to [31] and references therein), but perline ATP constraints are met here by properly choosing a transmit PSD value constant over all the lines/frequency bands (Sect. V).

The RAU average power constraint is fulfilled by introducing the scaling factor $\gamma$ in (12) such that it acts as a global gain scaling the power of the RAU transmit signal to meet the constraint. From (9) and (12), the constraint (3) can be re-framed as

$$
\begin{aligned}
\mathrm{E}\left[\left\|\mathbf{x}_{w}^{\mathrm{RAU}}\right\|_{2}^{2}\right] & =\mathrm{E}\left[\operatorname{tr}\left(\mathbf{x}_{w}^{\mathrm{RAU}} \mathbf{x}_{w}^{\mathrm{RAU}, H}\right)\right] \\
& =\gamma^{2} \operatorname{tr}\left(\mathbf{H}_{S F} \mathbf{F} \mathbf{F}^{H} \mathbf{H}_{S F}^{H}+\sigma_{c}^{2} \mathbf{I}\right) \leq N
\end{aligned}
$$

and $\gamma=\gamma_{\max }$, where $\gamma_{\max }$ follows from the equality in (19).

To tackle the PLPC power constraints at the cable input, it is convenient to decompose the precoding operator $\mathbf{F}$ at the BBU as follows:

$$
\mathbf{F}=\tilde{\mathbf{F}} \frac{1}{\beta}
$$

where $\tilde{\mathbf{F}}$ is the precoding matrix and $\beta$ enforces the power constraints (10). These yield

$$
\begin{array}{r}
\mathrm{E}\left[\left|\left[\mathbf{x}_{c, f}\right]_{j}\right|^{2}\right]=\frac{1}{\beta^{2}} \mathrm{E}\left[\left|\tilde{\mathbf{f}}_{f j}^{T} \mathbf{s}\right|^{2}\right]=\frac{1}{\beta^{2}}\left\|\tilde{\mathbf{f}}_{f j}^{T}\right\|_{2}^{2} \leq 1 \\
\forall f=1, \ldots, N_{f}, \forall j=1, \ldots, N_{s},
\end{array}
$$

where $\tilde{\mathbf{f}}_{f j}^{T}$ is the row of the matrix $\tilde{\mathbf{F}}$ which precodes the signal to be relayed over the $j$-th twisted-pair of the $f$-th cable frequency band. From (21) it follows that the PLPC power constraints are fulfilled by choosing $\beta$ as

$$
\beta^{2}=\left\|\tilde{\mathbf{F}}^{T}\right\|_{2, \infty}^{2}
$$

where the mixed norm $\|\mathbf{A}\|_{2, \infty}$ denotes the maximum column $l_{2}$-norm of matrix $\mathbf{A}$.

\section{BASE BAND UNIT PRECODING}

In A-MIMO-RoC all the computationally complex digital signal processing is shifted to the BBU, which performs an overall (digital) precoding of the end-to-end wired-pluswireless cascade channel from the BBU to all the intended UEs. Wireless and cable channels are assumed perfectly known at the BBU, as channel estimation techniques and precoder design under imperfect channel state information are beyond the scope of this paper.

To describe this overall signal model, let us gather the received signals as

$$
\mathbf{y}=\mathbf{H}_{w} \mathbf{x}_{w}^{\mathrm{RAU}}+\mathbf{n}_{w},
$$

where vector $\mathbf{y}=\left[y_{1}, y_{2}, \ldots, y_{N_{r}}\right]^{T}$ contains the signals received by all the $N_{r}$ UEs, $\mathbf{H}_{w}=$ $\left[\mathbf{h}_{w, 1}, \mathbf{h}_{w, 2}, \ldots, \mathbf{h}_{w, N_{r}}\right]^{T} \in \mathbb{C}^{N_{r} \times N}$ is the compound wireless channel matrix whose $k$-th row is the vector channel from the RAU to UE $k$-th, and $\mathbf{n}_{w}$ is the additive white Gaussian noise vector. Therefore, equation (23) becomes

$$
\mathbf{y}=\mathbf{H}_{\pi} \mathbf{F s} \cdot \gamma+\mathbf{n},
$$

where $\mathbf{H}_{\pi}=\mathbf{H}_{w} \mathbf{H}_{S F} \in \mathbb{C}^{N_{r} \times N}$ is the cascade of the permuted wired $\left(\mathbf{H}_{S F}\right)$ and wireless $\left(\mathbf{H}_{w}\right)$ channels with the subindex $\pi$ empathizing the dependence on the specific SF2SF multiplexing operator $\boldsymbol{\Pi}_{c}$, and vector $\mathbf{n}=\left[n_{1}, n_{2}, \ldots, n_{N_{r}}\right]^{T}$ collects the noise samples at all the receivers with $n_{k} \sim \mathcal{C N}\left(0, \gamma^{2} \sigma_{c}^{2}\left\|\mathbf{h}_{w, k}\right\|_{2}^{2}+\sigma_{w}^{2}\right)$ denoting the noise at the $k$-th UE.

As BBU-side processing, in this work we consider ZeroForcing $(\mathrm{ZF})$ precoding which forces to zero all the interference among the $N_{r}$ UEs. The precoding $\mathbf{F}$ is designed to fully diagonalize the compound wired-plus-wireless channel $\mathbf{H}_{\pi}$ to obtain an equivalent channel [32]

$$
\mathbf{H}_{e}=\mathbf{H}_{\pi} \mathbf{F}=\operatorname{diag}\left(h_{e, 1}, h_{e, 2}, \ldots, h_{e, N_{r}}\right),
$$

where here $h_{e, k}$ denotes the equivalent channel gain from the $\mathrm{BBU}$ to the $k$-th UE. This equivalent channel $h_{e, k}$ depends either on $\mathbf{F}$ and on the specific SF2SF multiplexing matrix $\boldsymbol{\Pi}_{c}$.

Directly from (24), it follows that for ZF precoding the decision variable at the $k$-th UE simplifies to

$$
y_{k}=\gamma h_{e, k} s_{k}+n_{k} \text {. }
$$

The corresponding UE rate $r_{k}$ is conventionally computed as

$$
r_{k}=\log _{2}\left(1+\frac{\left|h_{e, k}\right|^{2}}{\sigma_{c}^{2}\left\|\mathbf{h}_{w, k}\right\|_{2}^{2}+\sigma_{w}^{2} / \gamma^{2}}\right),
$$

and the sum-rate for all the UEs yields

$$
R=\sum_{k=1}^{N_{r}} r_{k}
$$

In the following, both linear and non-linear $\mathrm{ZF}$ precoding approaches are reviewed and adapted to the A-MIMO-RoC scenario. 


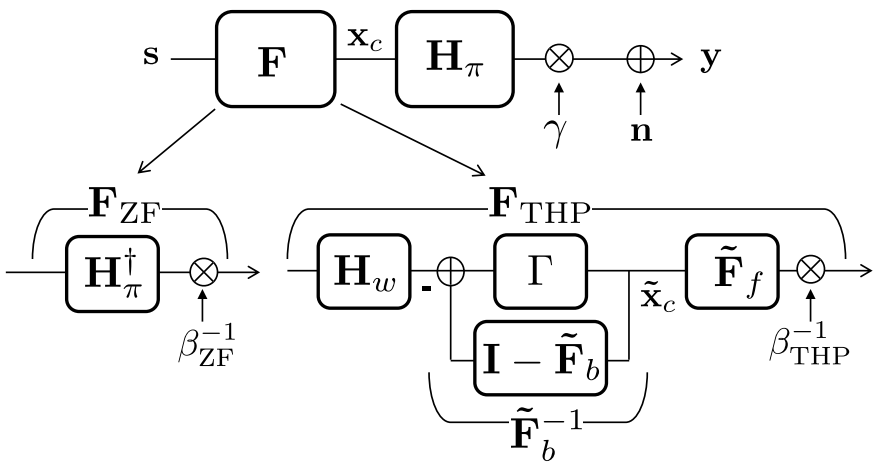

Fig. 3. BBU precoding schemes: linear Zero-Forcing (ZF) and TomlinsonHarashima Precoding (THP).

\section{A. Linear Zero Forcing}

In linear $\mathrm{ZF}$ the compound cascade of wired-plus-wireless channel $\mathbf{H}_{\pi}$ is compensated by the linear transformation

$$
\tilde{\mathbf{F}}_{\mathrm{ZF}}=\mathbf{H}_{\pi}^{\dagger} \text {, }
$$

where $\mathbf{A}^{\dagger}=\mathbf{A}^{H}\left(\mathbf{A} \mathbf{A}^{H}\right)^{-1}$ denotes the right Moore-Penrose pseudo-inverse of matrix $\mathbf{A}$. It is easy to prove that the $\mathrm{ZF}$ condition (25) is satisfied and the equivalent channel matrix is

$$
\mathbf{H}_{e, \mathrm{ZF}}=\mathbf{H}_{\pi} \mathbf{F}_{\mathrm{ZF}}=\frac{1}{\beta_{\mathrm{ZF}}} \mathbf{I},
$$

where $\beta_{\mathrm{ZF}}$ is computed by (22).

\section{B. Tomlinson-Harashima Precoding}

Linear ZF precoding usually suffers from a severe power increment due to the hard-inversion of the channel matrix. To overcome the problem, here we propose to employ Tomlinson-Harashima Precoding (THP), which is a non-linear Successive Interference Cancellation (SIC) precoding scheme, conventionally based on ZF criterion [33]. The THP block scheme is depicted in Fig. 3, where the precoding matrix $\tilde{\mathbf{F}}$ is decomposed into two sub-matrices as

$$
\tilde{\mathbf{F}}_{\mathrm{THP}}=\tilde{\mathbf{F}}_{f} \tilde{\mathbf{F}}_{b}^{-1},
$$

where $\tilde{\mathbf{F}}_{f} \in \mathbb{C}^{N \times N_{r}}$ is a linear matrix with orthonormal columns, usually referred to as feed-forward filter, and $\tilde{\mathbf{F}}_{b} \in$ $\mathbb{C}^{N_{r} \times N_{r}}$ is a lower-triangular matrix with units along the main diagonal, referred to as feed-back filter. The key characteristic which makes THP an attractive solution for applications with strict transmit power constraints is that the inversion of the triangular matrix $\tilde{\mathbf{F}}_{b}$ is performed by backward substitution together with a non-linear modulo operator $\Gamma$ (see Fig. 3), which limits the power of the encoded symbols within the boundaries of the original signal constellation, hence avoiding the typical noise enhancement which is the main performance limitation of ZF-based techniques.

Feed-forward filter $\tilde{\mathbf{F}}_{f}$ and feedback filter $\tilde{\mathbf{F}}_{b}$ are obtained by the QR decomposition of the compound channel matrix $\mathbf{H}_{\pi}$ as [34]

$$
\mathbf{H}_{\pi}{ }^{H}=\mathbf{Q R}=\left[\mathbf{Q}_{1}, \mathbf{Q}_{2}\right]\left[\begin{array}{c}
\mathbf{R}_{1} \\
\mathbf{0}
\end{array}\right]
$$

then

$$
\tilde{\mathbf{F}}_{f}=\mathbf{Q}_{1}, \quad \tilde{\mathbf{F}}_{b}=\operatorname{diag}\left(\mathbf{R}_{1}\right)^{-1} \mathbf{R}_{1}^{H},
$$

where $\mathbf{Q}_{1} \in \mathbb{C}^{N \times N_{r}}$ contains the first $N_{r}$ orthonormal columns of the unitary matrix $\mathbf{Q} \in \mathbb{C}^{N \times N}, \mathbf{R}_{1} \in \mathbb{C}^{N_{r} \times N_{r}}$ is an upper triangular matrix and $\operatorname{diag}\left(\mathbf{R}_{1}\right) \in \mathbb{C}^{N_{r} \times N_{r}}$ is a diagonal matrix that contains the main diagonal entries of $\mathbf{R}_{1}$. It is easy to show that the ZF condition (25) is satisfied and the equivalent channel matrix simplifies to

$$
\mathbf{H}_{e, \mathrm{THP}}=\mathbf{H}_{\pi} \mathbf{F}_{\mathrm{THP}}=\operatorname{diag}\left(\mathbf{R}_{1}\right) / \beta_{\mathrm{THP}} .
$$

Remark 1: In THP, the encoded symbols $\tilde{\mathbf{x}}_{c}$ at the output of the modulo operator (see Fig. 3) show a power increment with respect to the input symbols as $\sigma_{\tilde{x}}^{2}=\frac{M}{M-1} \sigma_{s}^{2}$, where $M$ is the size of the $M$-QAM signal constellation. However, this term becomes negligible for high modulation formats such as 256-QAM of LTE and its maximum value is $1.25 \mathrm{~dB}$ for smaller constellations such as BPSK [31], hence it has been neglected here.

\section{User Ordering Optimization}

The UE signals encoding order in the SIC procedure of THP strongly impacts the performance for all the UEs [35]. Such ordering operation is part of the possible row permutation of the compound wired-plus-wireless channel as $\overline{\mathbf{H}}_{\pi}=\boldsymbol{\Pi}_{w} \mathbf{H}_{\pi}$, where $\boldsymbol{\Pi}_{w} \in\{0,1\}^{N_{r} \times N_{r}}$ is another permutation matrix (not to be confused with cable mapping $\boldsymbol{\Pi}_{c}$ ) which is used prior to the feedback filter to enforce the desired UE encoding order. Permutations $\boldsymbol{\Pi}_{w}$ should be carefully chosen to optimize the desired performance metric (e.g., system sum-rate or minimum UE rate). However, an exhaustive search over all the possible $N$ ! channel permutations is unpractical, especially for a large number of UEs. Instead, in this work we incorporate the computation of the UE ordering matrix $\Pi_{w}$ in the $\mathrm{QR}$ procedure of THP with no additional computational complexity with respect to conventional non-ordered (i.e., $\boldsymbol{\Pi}_{w}=\mathbf{I}$ ) THP. Ordering has no impact on the system sum-rate (see Appendix A), hence, we consider here the minimum-rate maximization heuristic ordering algorithm proposed in [36] for DSL systems, denoted therein as Worst First (WF). For more details about ordering algorithms we refer the readers to [36] and references therein.

\section{Space-Frequency to Space- FREQUENCY MULTIPLEXING}

In this section, we detail the downlink SF2SF multiplexing optimization technique by first considering a simple singleUE downlink system, as it provides useful insights into the gain provided by the SF2SF technique alone without being affected by multi-user processing techniques such as FEXT mitigation or IL compensation. Following the approach in [23], it is first shown that the SF2SF problem for the single-UE Multiple-Input Single-Output case (SU-MISO) is equivalent the maximum Quadratic Assignment Problem (QAP), that is an NP-hard problem for which there is no known polynomialtime solving algorithm. Starting from these considerations, we detail an efficient heuristic algorithm for SU-MISO downlink SF2SF problem, which is later extended to the multi-user 
case by optimizing both the SF2SF multiplexing matrix $\Pi_{c}$ and the UE ordering matrix $\boldsymbol{\Pi}_{w}$, together with the design of the precoding matrix $\mathbf{F}$.

\section{A. SF2SF Problem for SU-MISO System}

The end-to-end capacity of the A-MIMO-RoC architecture is derived below for the SU-MISO case by setting the power scaling factors as $\gamma=\beta=1$, as for the single-UE case all power constraints are satisfied. From (24), the single-UE received signal yields

$$
y=\mathbf{h}^{H} \mathbf{f} s+n,
$$

where $\mathbf{h}^{H}=\mathbf{h}_{w}^{H} \mathbf{H}_{S F}$ is the cascade of radio $\left(\mathbf{h}_{w}\right)$ and cable $\left(\mathbf{H}_{S F}=\boldsymbol{\Pi}_{c}^{T} \mathbf{H}_{c} \boldsymbol{\Pi}_{c}\right)$ channels, and $\mathbf{f}=\mathbf{h} /\|\mathbf{h}\|_{2}$ is the conventional MISO beamforming for the overall Gaussian noise at the BBU $n \sim \mathcal{C N}\left(0, \sigma_{n}^{2}\right)$ with $\sigma_{n}^{2}=\left\|\mathbf{h}_{w}\right\|_{2}^{2} \sigma_{c}^{2}+\sigma_{w}^{2}$. Hence, (35) simplifies to $y=\|\mathbf{h}\|_{2} s+n$, and the corresponding channel capacity yields

$$
C=\log _{2}\left(1+\frac{\|\mathbf{h}\|_{2}^{2} \sigma_{s}^{2}}{\sigma_{n}^{2}}\right),
$$

where $\sigma_{s}^{2}=1$ by assumption and $\|\mathbf{h}\|_{2}^{2}=\mathbf{h}^{H} \mathbf{h}=\operatorname{tr}\left(\mathbf{h} \mathbf{h}^{H}\right)$.

Finally, the end-to-end capacity for a given radio channel realization $\mathbf{h}_{w}$ yields

$$
C\left(\boldsymbol{\Pi}_{c}\right)=\log _{2}\left(1+\left(\left\|\mathbf{h}_{w}\right\|_{2}^{2} \sigma_{c}^{2}+\sigma_{w}^{2}\right)^{-1} \cdot \Gamma\left(\boldsymbol{\Pi}_{c}\right)\right),
$$

which depends on the SF2SF mapping $\Pi_{c}$ and

$$
\Gamma\left(\boldsymbol{\Pi}_{c}\right)=\operatorname{tr}\left(\boldsymbol{\Pi}_{c}^{T} \mathbf{H}_{c} \mathbf{H}_{c}^{H} \boldsymbol{\Pi}_{c} \mathbf{h}_{w} \mathbf{h}_{w}^{H}\right)
$$

is the signal power at the decision variable. Alternatively, eq. (37) can be directly computed from (27) by noticing that for the SU-MISO case the equivalent channel is $h_{e, k}=\|\mathbf{h}\|_{2}$.

1) SF2SF Problem Formulation: For the SU-MISO case, the optimal SF2SF multiplexing is defined as that specific permutation matrix $\Pi_{c}$ that maximizes the single-UE end-toend capacity $C\left(\boldsymbol{\Pi}_{c}\right)$ in (37). As the function $\log _{2}(1+x)$ is a monotonic increasing function of $x$, maximizing (37) is equivalent to maximize its argument $\Gamma\left(\boldsymbol{\Pi}_{c}\right)$ (38), which simplifies the analysis here. This leads to the following nonlinear integer program:

$$
\begin{aligned}
\max _{\boldsymbol{\Pi}_{c}} & \Gamma\left(\boldsymbol{\Pi}_{c}\right) \\
\text { s. t. } & \sum_{n=1}^{N} p_{j f}^{n}=1, \forall f=1, \ldots, N_{f}, \forall j=1, \ldots, N_{s}, \\
& \sum_{j=1}^{N_{s}} \sum_{f=1}^{N_{f}} p_{j f}^{n}=1, \forall n=1, \ldots, N,
\end{aligned}
$$

where $p_{j f}^{n} \in\{0,1\} \forall f, j, n$, is the $n$-th entry of the row of matrix $\Pi_{c}$ responsible for the signal mapping over the $j$-th pair at the $f$-th band as defined in Sect. II-C, and the constraints ensure that each symbol is mapped onto only 1 SF channel and, symmetrically, each SF channel carries only 1 symbol (i.e., $\Pi_{c} \in \mathcal{P}$, with $\mathcal{P}$ denoting the set of permutation matrices).
The downlink SF2SF problem (39) reduces to the maximum QAP, which is a NP-hard combinatorial optimization problem [37], [38]. In particular, no exact algorithm can solve QAP problems of size $N>20$ in reasonable computational time, and even finding a locally optimal solution of the QAP can be prohibitively hard, i.e., no local criteria are known for deciding how good a locally optimal solution is as compared to a global one [39]. Moreover, although many techniques have been proposed to linearize the QAP [37], [40], these require several auxiliary variables and constraints that make it difficult to efficiently get the solution. These considerations pushed us to explore for an heuristic algorithm shaped to SF2SF that suboptimally solves (39) at low computational-complexity.

2) An Hill-Climbing Approach to the SF2SF Problem: A suboptimal solution to (39) can be found through an iterative local search with relatively low computational cost even when the number of RAU antennas $N$ grows up to several tens. The suboptimal SF2SF multiplexing by using an hill-climbing approach is an heuristic iterative optimization technique adapted from artificial intelligence problems [41].

The conventional hill-climbing procedure adapted for the SF2SF problem is based on the concept of neighboring permutations set: given an arbitrary permutation matrix $\Pi_{c}$, univocally defined by a vector $\boldsymbol{\pi}=\left[\pi_{1}, \ldots, \pi_{N}\right]^{T}$ as in Sect. II-C, the neighboring permutations set at distance $d_{\pi}$ is the set containing all vectors obtained by permuting in any way all subsets of $d_{\pi}$ elements in $\pi$. The algorithm starts from an initial, randomly chosen, permutation $\boldsymbol{\pi}_{0}$ and updates the solution as follows: at the $k$-th step all the neighbors $\left\{\boldsymbol{\pi}_{\text {near }, k}\right\}$ at distance lower than or equal to $d_{\pi}$ from the present solution $\pi_{\text {best, } k}$ are computed, together with the corresponding values of the signal power at the decision variable $\Gamma(\boldsymbol{\pi})$. The best neighbor, i.e., $\boldsymbol{\pi}_{\text {near }, k}^{\text {best }}=\arg \max \Gamma\left(\boldsymbol{\pi}_{\text {near }, k}\right)$, is obtained and compared to $\pi_{\text {best }, k}$ in terms of associated signal power. If $\Gamma\left(\boldsymbol{\pi}_{\text {near }, k}^{\text {best }}\right)>\Gamma\left(\boldsymbol{\pi}_{\text {best }, k}\right)$, the solution is updated to $\boldsymbol{\pi}_{\text {best }, k+1}=\boldsymbol{\pi}_{\text {near }, k}^{\text {best }}$ and another iteration is performed, otherwise the algorithm stops and the final solution is $\pi_{\text {best }, k}$. The total number of iterations $N_{\text {it }}$ needed to reach the solution depends both on the radio SNR $1 / \sigma_{w}^{2}$ and on the number of RAU antennas $N$, but it cannot be known in advance and needs to be evaluated numerically. The hill-climbing algorithm is clearly suboptimal as it selects as solution the first local maximum of the objective. Nevertheless, Sect. V shows that the loss with respect to exact solution of (39) is small.

Remark 2: In this work, we adopt the hill-climbing approach due to its simplicity and low number of iterations required to reach the solution. Nevertheless, other suboptimal algorithms may be used to solve the SF2SF problem, i.e., construction methods, tabu search, simulated annealing, genetic algorithms, greedy randomized adaptive search, ant colony systems, etc., each of which with its strengths and weaknesses [41]. For example, simulated annealing is very similar to the hill-climbing, but may prevent to get stuck in a local optimum at the expenses of a higher number of required iterations. A comprehensive comparison of iterative optimization techniques is out of the scope of the paper and is left as future works. 


\section{B. SF2SF Problem for MU-MISO System}

For the Multi-User MISO (MU-MISO) scenario considered in this section, two different SF2SF optimization problems are exploited: i) sum-rate maximization problem maximizing the overall system performance as

$$
\max _{\boldsymbol{\Pi}_{c} \in \mathcal{P}} R\left(\boldsymbol{\Pi}_{c}\right)=\sum_{k=1}^{N r} r_{k}\left(\mathbf{\Pi}_{c}\right),
$$

where $r_{k}$ is the rate for the $k$-th UE as defined in (27); ii) min-rate maximization problem maximizing the minimum rate among the UEs defined as

$$
\max _{\boldsymbol{\Pi}_{c} \in \mathcal{P}} \min _{k} r_{k}\left(\boldsymbol{\Pi}_{c}\right)
$$

As for the SU-MISO case, both the MU-MISO max-rate maximization (40) and min-rate maximization (41) SF2SF optimization problems are NP-hard. For the special case of $N=N_{r}$ (i.e., the number of UEs $N_{r}$ equals the number of antennas $N$ ) under THP design, this can be proved to follow similar steps as for SU-MISO systems (Sect. IV-A). However, in the general $N \geq N_{r}$ case, in MU-MISO scenario it is fairly complicated to derive analytically tractable expressions for the UE rate $r_{k}\left(\boldsymbol{\Pi}_{c}\right)$ and the sum-rate $R\left(\boldsymbol{\Pi}_{c}\right)$ due to the fact that both the scalar gains $\beta$ and $\gamma$ depend on the SF2SF mapping $\Pi_{c}$. Hence, MU-MISO considered here is for $N=N_{r}$ to provide the reader with an intuitive insight of the overall problem. In any case, the system design does not depend in any way by this assumption and it can be straightforwardly extended to the general $N \geq N_{r}$ case.

Problems (40) and (41) can be optimally solved only by an exhaustive search over all the possible $N$ ! permutations of the cable channel matrix $\mathbf{H}_{c}$, which is unpractical when the number of RAU antennas grows large (i.e., $N \gg 10$ ). As a low-complexity heuristic solution to the problem, we adopt the hill-climbing approach generalized to the MU-MISO case. The generalized hill-climbing algorithm is reported in Algorithm 1, where the precoding matrix $\tilde{\mathbf{F}}$ can be designed following both the linear ZF (Sect. III-A) or the THP (either ordered or not, Sect. III-B) criteria, and the objective function $f(\boldsymbol{\pi})$ can be either the sum-rate in (40) or the minimum UE rate in (41). Recall that UEs permutation $\Pi_{w}$ is accounted for separately.

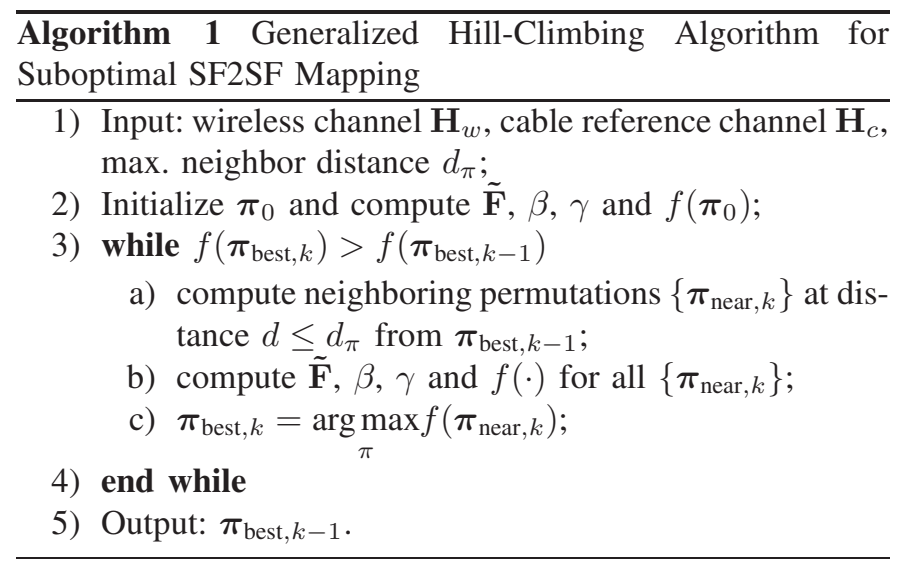

\section{Hill-Climbing Computational Complexity}

The evaluation of the optimal SF2SF multiplexing $\boldsymbol{\Pi}_{c}$ matrix follows from the positions of UEs by the directional radio channel $\mathbf{H}_{w}$, and, for each specific setting, the choice of $\Pi_{c}$ remains unchanged. In perspective, a number of possible configurations between UEs' positions and corresponding optimal SF2SF permutation matrices can be pre-computed and indexed when needed. This follows a learning approach of the context (not covered here) which represents an obvious solution for practical implementations.

In any case, the hill-climbing algorithm should be run for each UEs configuration, and its computational complexity can be measured by the total number of times, denoted by $K$, that the specific objective function (e.g., $\Gamma\left(\boldsymbol{\Pi}_{c}\right)$ for SU-MISO or $f\left(\boldsymbol{\Pi}_{c}\right)$ in Algorithm 1) needs to be evaluated in order to reach the solution. In the case of the SF2SF multiplexing by exhaustive search, for example, it corresponds to the total number of possible reference channel permutations: $K_{\text {exh }}=N$ !. The computational complexity of the hill-climbing algorithm needs to account for the fact that, for each of the $N_{\text {it }}$ hill-climbing iterations, an exhaustive search over all the neighboring permutations must be performed according to the neighboring distance $d_{\pi}$ and the cardinality of the neighboring permutations set $K_{d_{\pi}}$ :

$$
K_{d_{\pi}}=\sum_{n=2}^{d_{\pi}}\left(\begin{array}{l}
N \\
n
\end{array}\right) \cdot ! n,
$$

where $! n=n ! \cdot \sum_{i=0}^{n} \frac{(-1)^{i}}{n !}$ is known as derangement number (or de Montmort number), which is defined as the number of permutations of the elements of a set, such that no element appears in its original position (details omitted for brevity). The overall computational complexity is thus

$$
K=K_{d_{\pi}} \cdot N_{\mathrm{it}} .
$$

Notice that Eq. (43) must be multiplied by $N$ ! if another exhaustive search is performed to find the optimal UE ordering matrix $\Pi_{w}$ in Sect. III-C.

\section{Numerical Results}

To validate the effectiveness of the proposed A-MIMO-RoC architecture based on LAN cables, here we perform numerical evaluation based on cable channel measurements from the IEEE 802.3 Ethernet Working Group [29]. The cable is a 4-pairs Cat-6 cable with length $100 \mathrm{~m}$ (we refer to Fig. 3 of [18] for channel measurements) as this is conventionally used as reference distance, the bandwidth/pair is $\approx 500 \mathrm{MHz}$, and FH link uses typical parameters such as $-140 \mathrm{dBm} / \mathrm{Hz}$ noise floor PSD and $-80 \mathrm{dBm} / \mathrm{Hz} /$ pair transmit PSD, which meets the $8 \mathrm{dBm}$ cable per-line ATP constraint [30] in (11) even if the whole $500 \mathrm{MHz}$ cable bandwidth is employed. The radio channel (4) toward each UE is assumed to have $L=3$ paths for all the UEs, while, per each radio channel, the azimuth AoDs $\phi_{k, l}$ and the elevation AoDs $\theta_{k, l}$ at the RAU have been assumed uniformly random distributed as $\phi_{k, l} \sim \mathcal{U}(0,2 \pi)$ and $\theta_{k, l} \sim \mathcal{U}(-\pi / 4, \pi / 4)$ for indoor multi-path. The performance are evaluated in terms 
of UE rate averaged over 1000 radio channel realizations. We assume that the frequency division over the cable is made by bands of $B_{w}=22 \mathrm{MHz}$, corresponding to the bandwidth of the considered RF signals (according to recent measurements on indoor deployments, it is reasonable to assume the wireless channel as frequency-flat within this range [42], [43]). Since the cable frequency response is not flat (Fig. 3 of [18] shows that for the 100-m Cat- 6 cable considered here the IL varies approx. $1.5 \mathrm{~dB}$ within $22 \mathrm{MHz}$ wireless carrier bandwidth), for the sake of numerical analysis, we consider the IL and FEXT coefficients of the $k$-th matrix $\mathbf{H}_{c, k}$ in (7) as the midpoint of the channel measurements over the $k$-th $22-\mathrm{MHz}$ frequency band $^{1}$ [18]. Unless otherwise explicitly stated, it is assumed in the following that to serve $N$ antennas at the RAU, the first $N_{f}=N / N_{s}$ 22-MHz frequency bands are allocated over the cable (i.e., the channels are allocated by increasing level of IL and FEXT), and all the $N_{s}=4$ twisted-pair spatial channels are always used. To make a practical example, a total bandwidth of approx. $5 \cdot 22=110 \mathrm{MHz}$ is needed over the 4-pairs to transport the signal for $N=20 \mathrm{RAU}$ antennas by $22 \mathrm{MHz} / \mathrm{ea}$. A maximum number of antennas $N=88$ is considered in this work, corresponding approx. to the usage of the whole $500 \mathrm{MHz}$ bandwidth over the copper cable. Sect. V-A describes the performance benchmarks used for the numerical results, which are presented in Sect. V-B for the SU-MISO case, and in Sect. V-C for the MU-MISO case.

\section{A. Performance Benchmarks}

The performance of the proposed A-MIMO-RoC architecture have been compared with the two following benchmarks for the same simulation settings described above.

1) A-MIMO-RoC Without SF2SF or UE Ordering: In this scheme, no SF2SF multiplexing or UE ordering are considered. The UE rates are computed by (27) for $\boldsymbol{\Pi}_{c}=\mathbf{I}$ and (for the MU-MISO case) $\Pi_{w}=\mathbf{I}$.

2) Optimum SVD Relay: The optimum SVD Relay [27] is a particular meaningful benchmark for the MU-MISO case due to the similarities with our scheme. In fact, both schemes: i) are designed for the downlink of a multi-user MIMO two-hop AF channel; ii) employ the same precoding scheme (i.e., THP), and iii) optimize UE ordering. The main difference from our proposed architecture is that in [27] the RAU is not constrained to be all-analog, and thus it can perform the SVD processing [27]. On the contrary, due to the analog RAU, for A-MIMO-RoC this is not feasible: all the processing is completely moved to the BBU-side, and the only possibility to face the impairments of the cable $\mathrm{FH}$ at the RAU is by the proposed SF2SF multiplexing. In other words, the two schemes are almost the same, with the only difference that one performs optimal SVD processing at the RAU, and the other SF2SF multiplexing. Furthermore, as the SVD Relay is designed following a sum-rate maximization criterion, while the proposed heuristic solves a min-rate maximization

\footnotetext{
${ }^{1}$ If one considers multiple smaller frequency bands within each $22-\mathrm{MHz}$ channel, the mismatch between channel coefficients and precoder (calculated from the midpoint over the entire 22-MHz band) may lead to some performance degradation. In this regards, one possibility may be to adopt a THP implementation robust to imperfect channel state information [18], [44], [45]
}

TABLE I

SU-MISO RATES [BPS/Hz]: OPTIMAL VS HILL-CLIMBING BY VARYING NEIGHBoring Distance $d_{\pi}, N=8$ RAU ANTENNAS, $N_{s}=2$ FREQUENCY BANDS AT 300 AND $500 \mathrm{MHz}$

\begin{tabular}{|c|c|c|c|c|c|c|c|}
\hline \multirow{2}{*}{ Technique } & \multicolumn{6}{|c|}{ Radio SNR, $\mathbf{1} \boldsymbol{\sigma}_{\boldsymbol{w}}{ }^{2}[\mathbf{d B}]$} & \multirow{2}{*}{$\begin{array}{c}\text { Complexity }(\boldsymbol{K}) \\
\text { at } \mathbf{1} / \boldsymbol{\sigma}_{\boldsymbol{w}}^{2}=\mathbf{2 5} \mathbf{~ d B}\end{array}$} \\
\cline { 2 - 8 } & $\mathbf{5}$ & $\mathbf{1 0}$ & $\mathbf{1 5}$ & $\mathbf{2 0}$ & $\mathbf{2 5}$ & $\mathbf{3 0}$ & 40320 \\
\hline SF2SF: optimal (exh. search) & 0.007 & 0.023 & 0.068 & 0.207 & 0.541 & 1.245 & $1.48\left(N_{i t}=1.49\right)$ \\
\hline SF2SF: hill-climb., $d_{\boldsymbol{\pi}}=4$ & 0.007 & 0.022 & 0.066 & 0.206 & 0.540 & 1.244 & 1148 \\
\hline SF2SF: hill-climb., $d_{\boldsymbol{\pi}}=2$ & 0.006 & 0.020 & 0.060 & 0.195 & 0.530 & 1.234 & $39\left(N_{i t}=1.38\right)$ \\
\hline w/o SF2SF & 0.005 & 0.015 & 0.046 & 0.141 & 0.382 & 0.933 & - \\
\hline
\end{tabular}

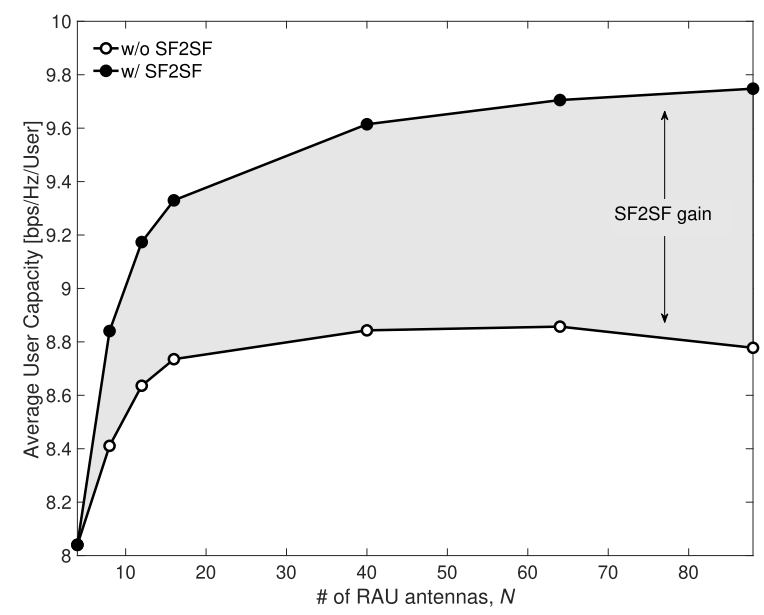

Fig. 4. SF2SF gain in SU-MISO: average UE rate vs number of RAU antennas $N(\approx N \cdot 22 \mathrm{MHz}$ bandwidth $), 1 / \sigma_{w}^{2}=25 \mathrm{~dB}$.

problem, it is necessary to compare on the same basis also the minimum and the maximum rates achieved by the two techniques. Further details on the SVD Relay scheme can be found in [27].

\section{B. SU-MISO with SF2SF}

In this section we make a few considerations about the SU-MISO case. First of all, in Table I the rates obtained by optimally and heuristically solving the SF2SF multiplexing problem are compared by varying the neighboring distance $d_{\pi}$, together with the computational complexity for a radio SNR $1 / \sigma_{w}^{2}=25 \mathrm{~dB}$ (used in the following as reference radio SNR). The parameter $N_{\mathrm{it}}$, evaluated numerically, is the average number of hill-climbing iterations needed to reach the solution. The $N$ ! computational complexity of the optimal solution forces us to consider here a relatively small problem instance: $N=8$ RAU antennas relay to the UE the BBU signals carried over $N_{f}=2$ cable bands at $300 \mathrm{MHz}$ and $500 \mathrm{MHz}$, to stress a condition where IL and FEXT are more severe [18]. Table I confirms that the hill-climbing approach leads to a solution which is quite close to the optimal one at much lower complexity: e.g., for $1 / \sigma_{w}^{2}=25 \mathrm{~dB}$ and $d_{\pi}=2$ (used as reference neighboring distance for all the following numerical evaluations) the gap from the optimum is $\approx 0.01 \mathrm{bps} / \mathrm{Hz}$, and this is achieved by performing only $K=39$ out of the total $K_{\text {exh }}=40,320$ searches required by the exhaustive search.

Fig. 4 shows the average UE rates with and without SF2SF multiplexing by progressively increasing the number of 
TABLE II

MU-Miso Rates [BPs/Hz] With Non-ORdered THP, Max Min-Rate Optimal SOLUTION VS Hill-Climbing B Y VARYing THE

Neighboring Distance $d_{\pi}, N=8$ RAU Antennas,

$N_{s}=2$ FREQUENCY BANDS AT 300 AND $500 \mathrm{MHz}$

\begin{tabular}{|c|c|c|c|c|c|c|c|}
\hline \multirow{2}{*}{ Technique } & \multicolumn{6}{|c|}{ Radio SNR, $1 / \sigma_{w}{ }^{2}[\mathrm{~dB}]$} & \multirow{2}{*}{$\begin{array}{c}\text { Complexity }(K) \\
\text { at } 1 / \sigma_{w}^{2}=25 \mathrm{~dB}\end{array}$} \\
\hline & 5 & 10 & 15 & 20 & 25 & 30 & \\
\hline SF2SF: optimal (exh. search) & 0.361 & 0.693 & 1.014 & 1.281 & 1.312 & 1.335 & 40320 \\
\hline SF2SF: hill-climb., $d_{\pi}=4$ & 0.355 & 0.689 & 1.008 & 1.274 & 1.307 & 1.329 & $1547\left(N_{i t}=2.01\right)$ \\
\hline SF2SF: hill-climb., $d_{\pi}=2$ & 0.321 & 0.652 & 0.976 & 1.225 & 1.255 & 1.266 & $64\left(N_{i t}=2.27\right)$ \\
\hline w/o SF2SF & 0.202 & 0.402 & 0.624 & 0.790 & 0.799 & 0.802 & - \\
\hline
\end{tabular}

antennas $N$ allocated on one cable (i.e., increasing the portion of allocated cable bandwidth) for a radio SNR $1 / \sigma_{w}^{2}=25 \mathrm{~dB}$. It is interesting to observe that the UE rate achieved without SF2SF starts to decrease around the $N=40 \mathrm{RAU}$ antennas ( $\approx 220 \mathrm{MHz}$ cable bandwidth): in fact, in order to serve more antennas, a higher frequency portion of the cable bandwidth should be allocated for which IL and FEXT are so severe that any improvement in the SINR at the decision variable becomes negligible. In other words, there is no point in using more antennas if cable excessively degrades the wireless rate and antennas are almost randomly multiplexed over the cable resources. On the contrary, the UE rate achieved with SF2SF multiplexing increases with the number of antennas/cable bandwidth. This confirms that, by means of an optimized cable resources management, SF2SF efficiently copes with the cable impairments enabling the usage of the available cable bandwidth with the UE rate improvement $>1 \mathrm{bps} / \mathrm{Hz}$. This is due to the fact that UE performance are mostly determined by a small subset of RAU antennas, which is not known in advance and depends on the specific UE configuration. SF2SF multiplexing selects and prioritizes the performance-sensitive antennas by assigning them SF cable channels for which cable impairments are less severe, thus creating virtual sub-arrays at the RAU that are minimally cable interfered. The remaining antennas are progressively assigned to cable SF resources.

\section{MU-MISO with SF2SF}

The performance of the A-MIMO-RoC architecture are evaluated here in presence of multiple single-antenna UEs, which mandatorily requires $\mathrm{BBU}$ precoding to mitigate the multi-user interference due to the cascade of wired and wireless channels. In this scenario, we assume that the number of served UEs $N_{r}$ equals the number of RAU antennas $N$, meaning that the more RAU antennas we employ, the more UEs we are able to serve but, on the other hand, the more bandwidth we need to allocate over the cable fronthaul (i.e., $N=N_{r}=N_{s} \cdot N_{f}$, with $N_{s}=4$ ).

As for the SU-MISO case, we first demonstrate that also in the MU-MISO scenario the performance obtained by the hillclimbing approach the optimal achieved by the $N$ ! exhaustive search. This is shown in Table II for the min-rate maximization SF2SF problem with non-ordered THP precoding, and assuming to employ $N=8 \mathrm{RAU}$ antennas forwarding to the UEs the BBU signals coming from $N_{f}=2$ cable frequency bands allocated similarly as SU-MISO at $300 \mathrm{MHz}$ and $500 \mathrm{MHz}$ (reduced problem size due to the complexity of the optimal solution). THP makes the rates larger than the ones reported in Table I.

A similar result is in Table III, which shows the UEs rates obtained by jointly solving both the min-rate maximization SF2SF and the min-rate maximization UE ordering problems. In particular, Table III compares $i$ ) the rates obtained by optimally solving both problems (i.e., by an exhaustive search over all the $N$ ! possible SF2SF permutations $\Pi_{c}$ combined with all the $N$ ! UE ordering permutations $\Pi_{w}$ ), ii) the rates obtained by the fully heuristic solution (i.e., hill-climbing algorithm plus WF ordering), and iii) the rates obtained by the two hybrid optimal-heuristic solutions (i.e., exhaustive search for SF2SF plus WF ordering and, vice versa, hillclimbing plus exhaustive search for UE ordering). Due to the $(N !)^{2}$ searches required by the optimal solution, only $N=6$ antennas have been employed here at RAU to relay the BBU signals carried over only $N_{s}=3$ out of the 4 pairs (one pair for other services) and $N_{f}=2$ cable bands allocated over the cable at $300 \mathrm{MHz}$ and $500 \mathrm{MHz}$. Table III shows that the fully heuristic solution achieves a good trade-off between performance (approx. $0.4 \mathrm{bps} / \mathrm{Hz}$ loss) and computational complexity (approx. 4 order of magnitude less expensive). It is worth noting that the nearly-optimal performance achieved by the combination of hill-climbing algorithm and optimal UE ordering are still prohibitive from a computational cost perspective.

The mean UE rate versus $1 / \sigma_{w}^{2}$ is shown in Fig. 5a for linear ZF precoding and non-ordered THP, with and without sum-rate maximization SF2SF mapping, and for $N=24$ RAU antennas. Beside the expected superior performance of THP with respect to linear ZF, it is worth noting that, for linear ZF, even if SF2SF multiplexing improves the mean UE rate by almost $33 \%$, the performance achieved for the considered scenario are very poor, i.e., slightly more then $2 \mathrm{bps} / \mathrm{Hz}$ for $1 / \sigma_{w}^{2}=25 \mathrm{~dB}$. As expected (see Appendix A), the impact of SF2SF on the mean UE rate of THP is almost indiscernible, which is the reason why for the remainder of the numerical results the min-rate maximization criterion is preferred to the sum-rate maximization one. The performance of THP are shown in Fig. 5b for $N=24$ RAU antennas with and without min-rate maximization SF2SF multiplexing, and with and without WF ordering. Again, the impact of both SF2SF and WF ordering on the mean UE rate is negligible, and all methods converge to the same rate as SNR increases. However, the minimum rate per UE around $1 / \sigma_{w}^{2}=25 \mathrm{~dB}$ is improved by $0.5 \mathrm{bps} / \mathrm{Hz}$ in the case of SF2SF alone and by more then $3 \mathrm{bps} / \mathrm{Hz}$ in the case of WF-ordered THP, while the gain with respect to conventional THP raises up to $\approx 5$ $\mathrm{bps} / \mathrm{Hz}$ when the two techniques are combined together.

The impact of SF2SF multiplexing on the performance of THP-based techniques is shown in Fig. 6 a for $1 / \sigma_{w}^{2}=25 \mathrm{~dB}$ by increasing the number of served UEs $N_{r}$, and, consequently, by increasing both the number of RAU antennas $N$ and the allocated cable bandwidth. It follows from Fig. 6a that the achieved mean UE rate (equal for all methods) is slightly more then $6 \mathrm{bps} / \mathrm{Hz}$ when serving all the $N_{r}=88$ UEs. Non-ordered THP with SF2SF (dotted-lines, cross-maker) achieves exactly the same maximum rate of conventional THP 
TABLE III

MU-Miso Rates [BPs/Hz] With Ordered ThP, Max Min-Rate Optimal Solution/Ordering vs Heuristics, $N=6 \mathrm{RAU}$ ANTENNAS, $N_{s}=2$ FREQUENCY BANDS AT $300 \mathrm{AND} 500 \mathrm{MHz}$

\begin{tabular}{|c|c|c|c|c|c|c|c|c|}
\hline \multirow{2}{*}{$\begin{array}{l}\text { Type of SF2SF } \\
\text { optimization }\end{array}$} & \multirow{2}{*}{$\begin{array}{c}\text { Type of UE ordering } \\
\text { optimization }\end{array}$} & \multicolumn{6}{|c|}{ Radio SNR, $1 / \sigma_{w}{ }^{2}[\mathrm{~dB}]$} & \multirow{2}{*}{$\begin{array}{r}\text { Complexity }(K) \\
\text { at } 1 / \sigma_{w}^{2}=25 \mathrm{~dB}\end{array}$} \\
\hline & & 5 & 10 & 15 & 20 & 25 & 30 & \\
\hline optimal (exh. search) & optimal (exh. search) & 0.856 & 1.462 & 2.053 & 2.376 & 2.600 & 2.620 & 518400 \\
\hline hill-climbing, $d_{\pi}=2$ & optimal (exh. search) & 0.852 & 1.458 & 2.048 & 2.371 & 2.597 & 2.601 & $23760\left(N_{i t}=2.2\right)$ \\
\hline optimal (exh. search) & WF ordering & 0.821 & 1.389 & 1.917 & 2.118 & 2.304 & 2.311 & 720 \\
\hline hill-climbing, $d_{\pi}=2$ & WF ordering & 0.784 & 1.346 & 1.865 & 2.059 & 2.236 & 2.241 & $32\left(N_{i t}=2.15\right)$ \\
\hline no & no & 0.189 & 0.494 & 0.766 & 0.987 & 1.118 & 1.148 & - \\
\hline
\end{tabular}

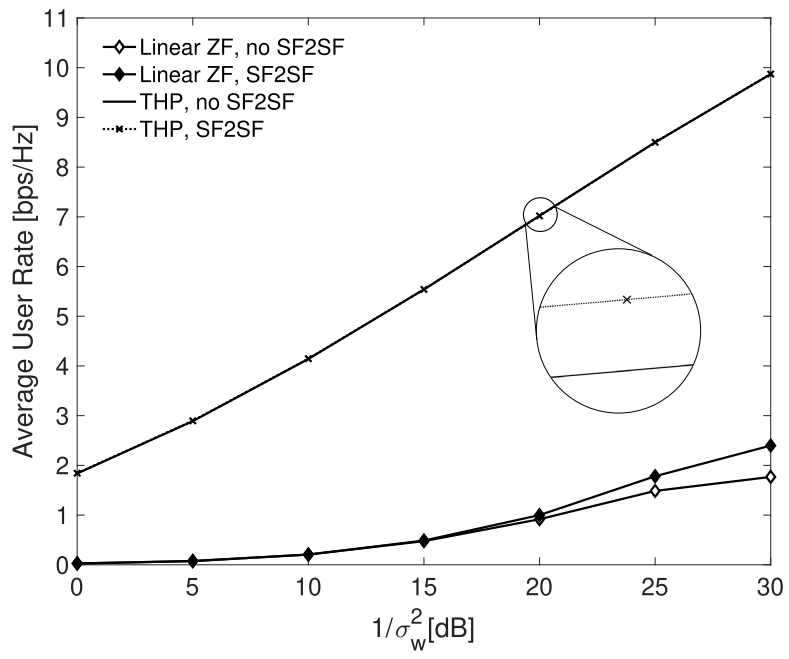

(a) Linear ZF vs THP w/ and w/o SF2SF (max sum-rate).

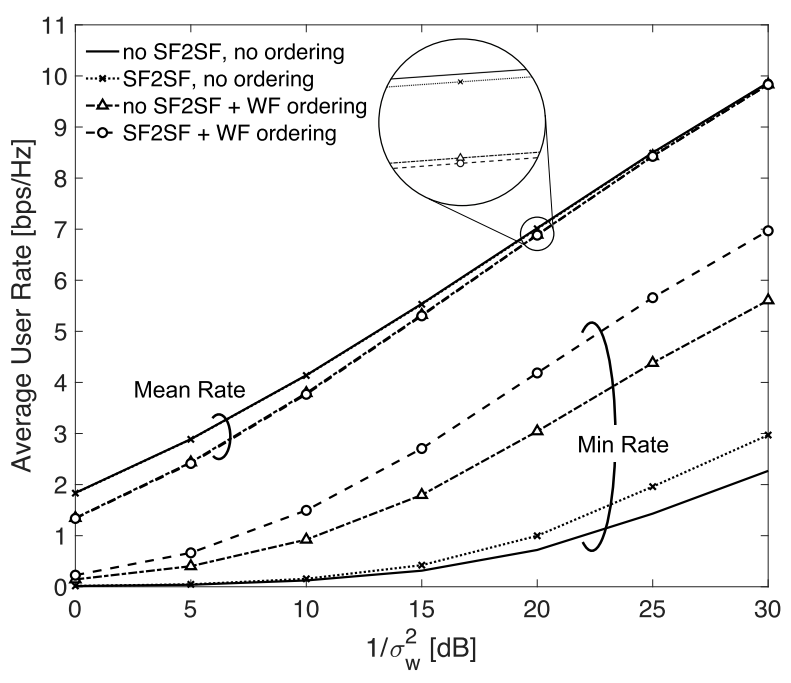

(b) THP w/ and w/o SF2SF and WF ordering (max min-rate).

Fig. 5. Average UE rate vs $1 / \sigma_{w}^{2}$ for $N=24 \mathrm{RAU}$ antennas $w /$ and w/o SF2SF multiplexing.

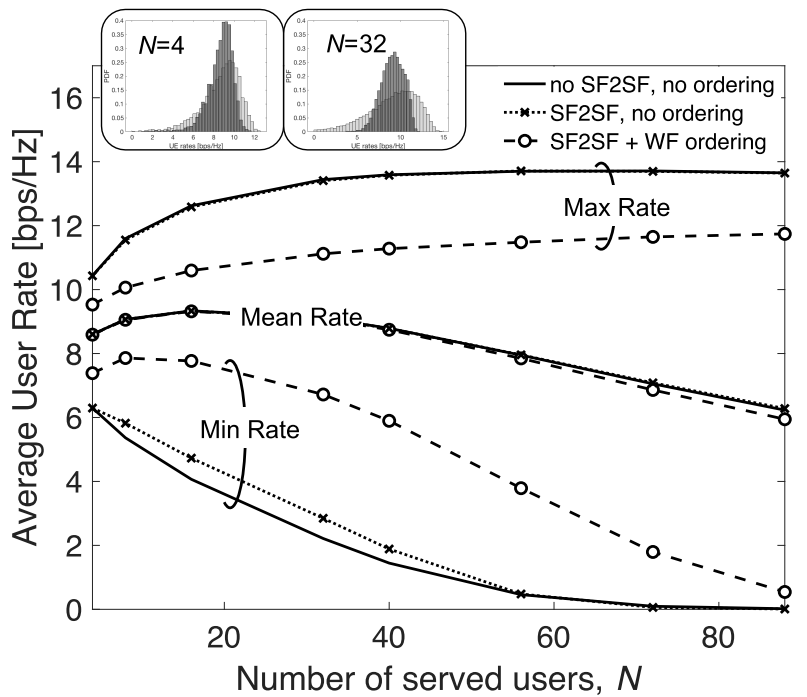

(a) Comparison of THP techniques w/ and w/o WF ordering and w/ and w/o maxmin rate $\mathrm{SF} 2 \mathrm{SF}$.

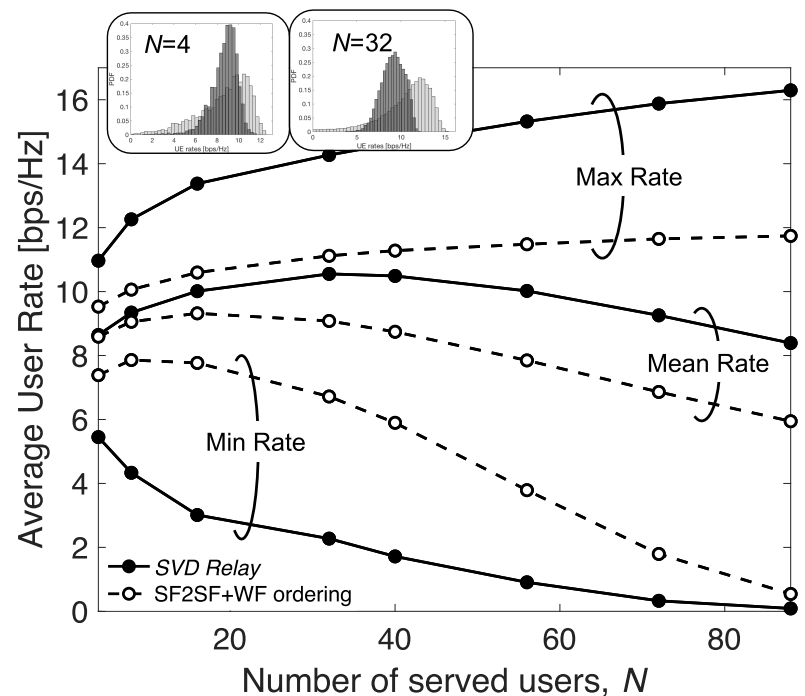

(b) WF-THP + SF2SF vs SVD Relay [27].

Fig. 6. MU-MISO for $1 / \sigma_{w}^{2}=25 \mathrm{~dB}$ : UEs rates vs RAU antennas $N$ and their distribution for "SF2SF+WF" (dark gray) vs $a$ ) "no SF2SF, no ordering" or $b$ ) "SVD Relay" (light gray).

(solid line) still improving the minimum. Again, the utmost gain in terms of minimum UE rate is achieved when SF2SF is combined with WF ordering (dashed-line, circle marker), which is able to provide $7 \mathrm{bps} / \mathrm{Hz}$ up to approx. $N_{r}=30$ UEs, while at least $6 \mathrm{bps} / \mathrm{Hz}$ and $4 \mathrm{bps} / \mathrm{Hz}$ are guaranteed for
$N_{r}=40$ and $N_{r}=56$ UEs, respectively. The minimum UE rate drops down to less then $2 \mathrm{bps} / \mathrm{Hz}$ only approaching the $N_{r}=70$ served UEs. The computational complexity required by the hill-climbing algorithm for the results in Fig. 6a is reported in Table IV, which shows how it scales with the 
TABLE IV

MU-MISO FOR $1 / \sigma_{w}^{2}=25$ DB: COMPUTATIONAL COMPLEXITY FOR THP MAX-Min RATE SF2SF MULTIPLEXING W/ AND W/O WF ORDERING

\begin{tabular}{|c|c|c|c|c|c|c|c|c|}
\hline \multirow{2}{*}{ Technique } & \multicolumn{7}{|c|}{ Number of RAU antennas $(\boldsymbol{N})$} \\
\cline { 2 - 9 } & $\mathbf{4}$ & $\mathbf{8}$ & $\mathbf{1 6}$ & $\mathbf{3 2}$ & $\mathbf{4 0}$ & $\mathbf{5 6}$ & $\mathbf{7 2}$ & $\mathbf{8 8}$ \\
\hline $\begin{array}{c}\text { SF2SF: optimal } \\
\text { (exh. search) }\end{array}$ & 24 & 40320 & $\approx 10^{13}$ & $\approx 10^{35}$ & $\approx 10^{47}$ & $\approx 10^{74}$ & $\approx 10^{103}$ & $\approx 10^{134}$ \\
\hline $\begin{array}{c}\text { SF2SF: hill-climb., } \\
d_{\pi}=2 \text {, no ordering }\end{array}$ & $\begin{array}{c}6 \\
\left(N_{i t}=1\right)\end{array}$ & $\begin{array}{c}70 \\
\left(N_{i t}=2.51\right)\end{array}$ & $\begin{array}{c}451 \\
\left(N_{i t}=3.76\right)\end{array}$ & $\begin{array}{c}2.21 \cdot 10^{3} \\
\left(N_{i t}=4.46\right)\end{array}$ & $\begin{array}{c}3 \cdot 10^{3} \\
\left(N_{i t}=3.95\right)\end{array}$ & $\begin{array}{c}2.63 \cdot 10^{3} \\
\left(N_{i t}=1.71\right)\end{array}$ & $\begin{array}{c}2.58 \cdot 10^{3} \\
\left(N_{i t}=1.01\right)\end{array}$ & $\begin{array}{c}3.83 \cdot 10^{3} \\
\left(N_{i t}=1\right)\end{array}$ \\
\hline $\begin{array}{c}\text { SF2SF: hill-climb., } \\
d_{\pi}=2 \text {, WF ordering }\end{array}$ & $\begin{array}{c}6 \\
\left(N_{i t}=1\right)\end{array}$ & $\begin{array}{c}67 \\
\left(N_{i t}=2.38\right)\end{array}$ & $\begin{array}{c}409 \\
\left(N_{i t}=3.41\right)\end{array}$ & $\begin{array}{c}2.16 \cdot 10^{3} \\
\left(N_{i t}=4.35\right)\end{array}$ & $\begin{array}{c}3.77 \cdot 10^{3} \\
\left(N_{i t}=4.84\right)\end{array}$ & $\begin{array}{c}8.07 \cdot 10^{3} \\
\left(N_{i t}=5.24\right)\end{array}$ & $\begin{array}{c}1.13 \cdot 10^{4} \\
\left(N_{i t}=4.41\right)\end{array}$ & $\begin{array}{c}7.5 \cdot 10^{3} \\
\left(N_{i t}=1.96\right)\end{array}$ \\
\hline
\end{tabular}
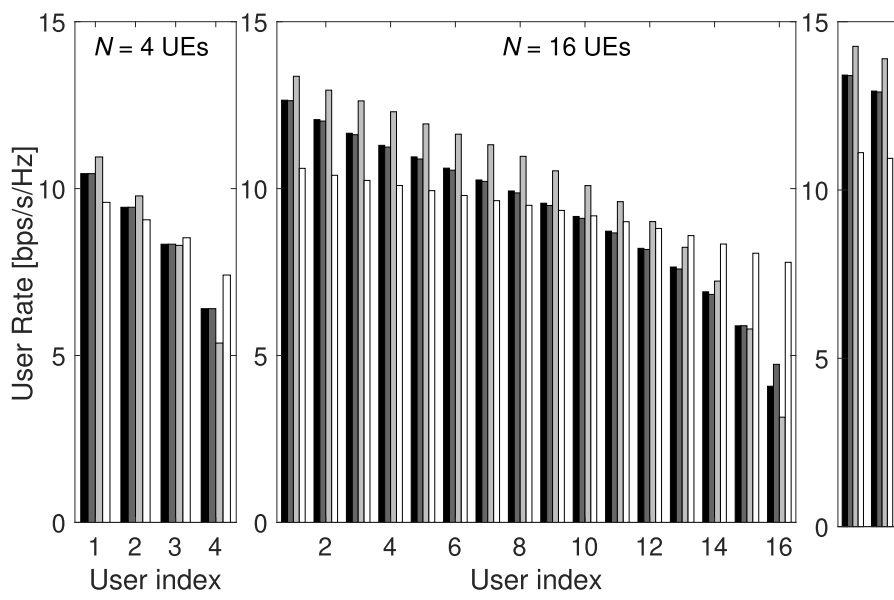

Fig. 7. MU-MISO for $1 / \sigma_{w}^{2}=25 \mathrm{~dB}$ : per-UE rate for $N=4,16,32$ UEs.

number of RAU antennas. The distribution of the UEs rates obtained with SF2SF-plus-WF (dark gray) has been compared with the one obtained by the baseline "no SF2SF, no ordering" (light gray) for $N=4,32$. This confirms that the proposed scheme reduces the dispersion of the UEs rates, thus raising the minimum rate at the expenses of a loss in the maximum rate.

Fig. 6b shows the comparison between THP with WF ordering and SF2SF multiplexing, which is the best-performing among the proposed methods, and the optimum SVD Relay scheme. It can be observed the gain achieved by the proposed technique in terms of minimum UE rate with respect to the one in [27], which is higher for a lower number of UEs (say < 40), while it reduces to few bps $/ \mathrm{Hz}$ approaching the 88 UEs. As expected, however, there is a price to pay in terms of mean and maximum UE rate for prioritizing minimum UE rate and for avoiding any signal processing at the RAU: this amounts to approx. $2 \mathrm{bps} / \mathrm{Hz}$ and $4 \mathrm{bps} / \mathrm{Hz}$ (for $N_{r}=$ 88 UEs) in mean and maximum UE rates, respectively. The distribution of the UEs rates obtained with SF2SF-plus-WF (dark gray) and the SVD Relay scheme (light gray) confirms the advantages of SF2SF allocation.

Finally, Fig. 7 shows for the same settings of Fig. 6 the rates for each of the UEs for $N=4,16,32$ RAU antennas (and thus number of UEs). Without SF2SF and UE ordering, the UEs rates present a decreasing behavior with the UE index (this is an inherent characteristics of THP-based techniques and is due to the SIC procedure [36]), which results in a large performance difference among UEs. On the contrary, our proposed technique provides a fairer performance distribution across UEs, hence raising the minimum guaranteed UE rate.

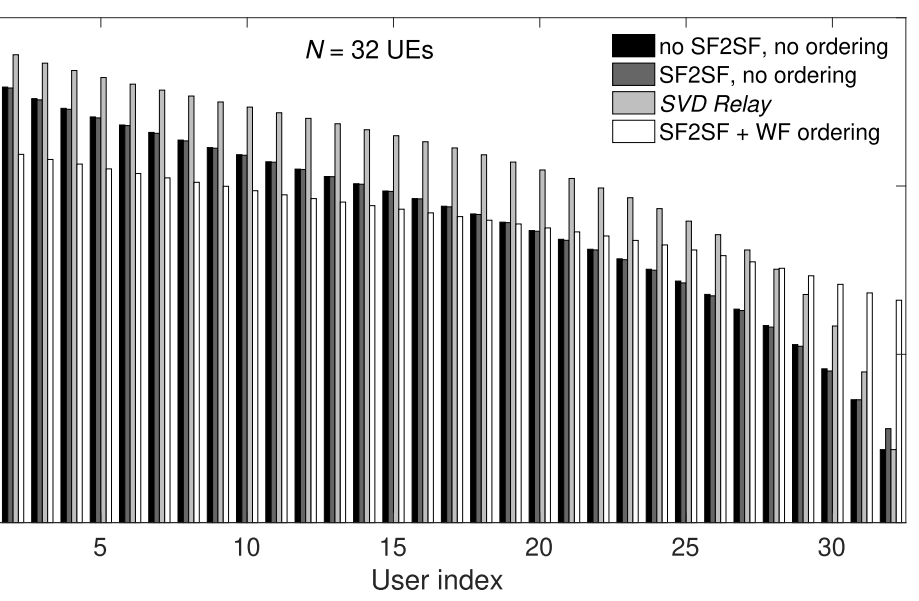

\section{CONCLUSions}

This paper investigates the downlink of the Analog MIMO Radio-over-Copper (A-MIMO-RoC) architecture, in which the overall channel from the BaseBand Unit (BBU) to the endusers is made by the cascade of a MIMO cable and a MIMO radio channels. The focus here is on the mapping between air- and cable-link resources, referred to as Space-Frequency to Space-Frequency (SF2SF) multiplexing. In particular, this paper shows that, in case of multiple-users served in the same physical resources, the optimization of SF2SF multiplexing, combined with digital-precoding at the BBU and user ordering, allows to cope with cable impairments providing substantial performance gains in terms of minimum rate guaranteed for all the users. Numerical results also show that the proposed architecture fairly compares with existing amplify-and-forward design techniques achieving an interesting trade-off between performance and overall system complexity, which makes A-MIMO-RoC an attractive solution for indoor applications.

\section{APPENDIX}

\section{A. Impact of SF2SF and UE Ordering on the A-MIMO-RoC Sum-Rate}

From (27) and (28), the sum-rate of the system can be computed as

$$
\begin{aligned}
R & =\sum_{k=1}^{N_{r}} \log _{2}\left(1+\frac{\left|h_{e, k}\right|^{2}}{\sigma_{c}^{2}\left\|\mathbf{h}_{w, k}\right\|_{2}^{2}+\sigma_{w}^{2} / \gamma^{2}}\right) \\
& \approx \log _{2}\left(\left|\mathbf{I}+\mathbf{R}_{n}^{-1} \mathbf{H}_{e} \mathbf{H}_{e}^{H}\right|\right) \approx \log _{2}\left(\left|\mathbf{R}_{n}^{-1} \mathbf{H}_{e} \mathbf{H}_{e}^{H}\right|\right),
\end{aligned}
$$


where $\mathbf{R}_{n}=\sigma_{c}^{2} \cdot \operatorname{diag}\left(\left\|\mathbf{h}_{w, 1}\right\|_{2}^{2},\left\|\mathbf{h}_{w, 2}\right\|_{2}^{2}, \ldots,\left\|\mathbf{h}_{w, N_{r}}\right\|_{2}^{2}\right)+$ $\mathbf{I} \frac{\sigma_{w}^{2}}{\gamma^{2}}$ is a diagonal matrix containing the equivalent noise variances at all the $N_{r}$ UEs, and $\mathbf{H}_{e}=\mathbf{H}_{\pi} \mathbf{F}$ is the equivalent channel matrix defined in (25) with $\mathbf{H}_{\pi}=\mathbf{H}_{w} \boldsymbol{\Pi}_{c}^{T} \mathbf{H}_{c} \boldsymbol{\Pi}_{c}$ and $\mathbf{F}=\beta^{-1}\left(\boldsymbol{\Pi}_{w} \mathbf{H}_{\pi}\right)^{-1}$ denoting the (permuted) wired-plus-wireless channel and the THP precoding matrix (see Sect. III-B), respectively. The approximation in (44) follows from the high-SNR regime of the systems.

For $N_{r}=N$, the equivalent channel $\mathbf{H}_{e}$ simplifies to $\mathbf{H}_{e}=\beta^{-1} \mathbf{H}_{w} \boldsymbol{\Pi}_{c}^{T} \mathbf{H}_{c} \boldsymbol{\Pi}_{c} \mathbf{H}_{\pi}^{-1} \boldsymbol{\Pi}_{w}^{T}$. Finally, by the determinant property $\left|\prod_{k} \mathbf{A}_{k}\right|=\prod_{k}\left|\mathbf{A}_{k}\right|$ and recalling that the determinant of unitary matrices is $\left|\boldsymbol{\Pi}_{w}\right|=\left|\boldsymbol{\Pi}_{c}\right|=1$, it is easy to prove that the impact of both the SF2SF multiplexing $\boldsymbol{\Pi}_{c}$ and the UE ordering $\boldsymbol{\Pi}_{w}$ is negligible on the sum-rate in (44), as shown in Sect. V.

In fact, from an information theoretical perspective, the wired-plus-wireless channel from the BBU to the UEs is a Gaussian Broadcast Channel (GBC), whose capacity is achieved by the Dirty Paper Coding (DPC) scheme [46]. As THP is a practical implementation of DPC, it is reasonable to assume that any THP-based technique is in principle capable to nearly achieve the GBC capacity (i.e., the sum-rate), regardless the users' ordering or the SF2SF multiplexing. In practice, as shown above, this condition is met for high-SNR.

\section{REFERENCES}

[1] 5G-Thriving Indoors, Cisco White Paper. [Online]. Available: https:// www.cisco.com/c/dam/en/us/solutions/collateral/service-provider/ultraservices-platform $/ 5 \mathrm{~g}$-ran-indoor.pdf.

[2] A. Checko et al., "Cloud RAN for mobile networks-A technology overview," IEEE Commun. Surveys Tuts., vol. 17, no. 1, pp. 405-426, 1st Quart., 2015.

[3] Ericsson AB, Huawei Technologies Co. Ltd., NEC Corp., Alcatel Lucent and Nokia Networks. (Sep. 2014). CPRI Specifications V.6.1 (2014-07-01). [Online]. Available: http://www.cpri.info/downloads/ CPRI_v_6_1_2014-07-01.pdf

[4] Study on New Radio Access Technology: Radio Access Architecture and Interfaces (Release 14), document TR 38.801 v14.0.0, 3GPP TSG RAN, Mar. 2017.

[5] D. Wübben et al., "Benefits and impact of cloud computing on 5G signal processing: Flexible centralization through cloud-RAN," IEEE Signal Process. Mag., vol. 31, no. 6, pp. 35-44, Nov. 2014.

[6] J. Bartelt, P. Rost, D. Wübben, J. Lessmann, B. Melis, and G. Fettweis, "Fronthaul and backhaul requirements of flexibly centralized radio access networks," IEEE Wireless Commun., vol. 22, no. 5, pp. 105-111, Oct. 2015.

[7] Y. Huang, C. Lu, M. Berg, and P. Ödling, "Functional split of zeroforcing based massive MIMO for fronthaul load reduction," IEEE Access, vol. 6, pp. 6350-6359, 2018.

[8] A. S. Thyagaturu, Z. Alharbi, and M. Reisslein, "R-FFT: Function split at IFFT/FFT in unified LTE CRAN and cable access network," IEEE Trans. Broadcast., vol. 64, no. 3, pp. 648-665, Sep. 2018.

[9] (Aug. 2017). eCPRI Press Release. http://www.cpri.info/press.html

[10] J. Gambini and U. Spagnolini, "Wireless over cable for femtocell systems," IEEE Commun. Mag., vol. 51, no. 5, pp. 178-185, May 2013.

[11] D. Wake, A. Nkansah, and N. J. Gomes, "Radio over fiber link design for next generation wireless systems," J. Lightw. Technol., vol. 28, no. 16, pp. 2456-2464, Aug. 15, 2010.

[12] J. Gambini and U. Spagnolini, "Radio over telephone lines in femtocell systems," in Proc. IEEE PIMRC, Sep. 2010, pp. 1544-1549.

[13] L. Combi, A. Gatto, M. Martinelli, P. Parolari, and U. Spagnolini, "Pulse-width optical modulation for CRAN front-hauling," in Proc. IEEE Globecom Workshops (GC Wkshps), Dec. 2015, pp. 1-5.

[14] C. Lu et al., "Connecting the dots: Small cells shape up for highperformance indoor radio," Ericsson Rev., vol. 91, no. 2, pp. 38-45, 2014.
[15] F. Tonini, M. Fiorani, M. Furdek, C. Raffaelli, L. Wosinska, and P. Monti, "Radio and transport planning of centralized radio architectures in 5G indoor scenarios," IEEE J. Sel. Areas Commun., vol. 35, no. 8, pp. 1837-1848, Aug. 2017.

[16] Y. Huang et al., "LTE over copper-Potential and limitations," in Proc. IEEE 26th Annu. Int. Symp. Pers., Indoor, Mobile Radio Commun. (PIMRC), Aug./Sep. 2015, pp. 1339-1343.

[17] E. Medeiros et al., "Crosstalk mitigation for LTE-over-copper in downlink direction," IEEE Commun. Lett., vol. 20, no. 7, pp. 1425-1428, Jul. 2016.

[18] S. H. R. Naqvi, A. Matera, L. Combi, and U. Spagnolini, "On the transport capability of LAN cables in all-analog MIMO-RoC fronthaul," in Proc. IEEE Wireless Commun. Netw. Conf. (WCNC), Mar. 2017, pp. $1-6$,

[19] A. Matera, V. Rampa, M. Donati, A. Colamonico, A. F. Cattoni, and U. Spagnolini. (2018). "A first experimental demonstration of analog MIMO radio-over-copper." [Online]. Available: https://arxiv.org/ abs/1809.05363

[20] A. Matera, L. Combi, S. H. R. Naqvi, and U. Spagnolini, "Spacefrequency to space-frequency for MIMO radio over copper," in Proc. IEEE Int. Conf. Commun. (ICC), May 2017, pp. 1-6.

[21] A. Matera and U. Spagnolini, "On the optimal space-frequency to frequency mapping in indoor single-pair RoC fronthaul," in Proc. Eur. Conf. Netw. Commun. (EuCNC), Jun. 2017, pp. 1-5.

[22] A. Matera, R. Kassab, O. Simeone, and U. Spagnolini, "Non-orthogonal eMBB-URLLC radio access for cloud radio access networks with analog fronthauling," Entropy, vol. 20, no. 9, p. 661, 2018.

[23] A. Matera and U. Spagnolini, "Analog MIMO-RoC downlink with SF2SF," IEEE Wireless Commun. Lett., vol. 8, no. 1, pp. 73-76, Feb. 2019.

[24] X. Tang and Y. Hua, "Optimal design of non-regenerative MIMO wireless relays," IEEE Trans. Wireless Commun., vol. 6, no. 4, pp. 1398-1407, Apr. 2007.

[25] O. Munoz-Medina, J. Vidal, and A. Agustin, "Linear transceiver design in nonregenerative relays with channel state information," IEEE Trans. Signal Process., vol. 55, no. 6, pp. 2593-2604, Jun. 2007.

[26] L. Sanguinetti, A. A. D'Amico, and Y. Rong, "A tutorial on the optimization of amplify-and-forward MIMO relay systems," IEEE J. Sel. Areas Commun., vol. 30, no. 8, pp. 1331-1346, Sep. 2012.

[27] C.-B. Chae, T. Tang, R. W. Heath, Jr., and S. Cho, "MIMO relaying with linear processing for multiuser transmission in fixed relay networks," IEEE Trans. Signal Process., vol. 56, no. 2, pp. 727-738, Feb. 2008.

[28] O. El Ayach, S. Rajagopal, S. Abu-Surra, Z. Pi, and R. W. Heath, Jr., "Spatially sparse precoding in millimeter wave MIMO systems," IEEE Trans. Wireless Commun., vol. 13, no. 3, pp. 1499-1513, Mar. 2014.

[29] IEEE P802.3an (10GBASE-T) Task Force. Accessed: Jan. 2019. [Online]. Available: http://www.ieee802.org/3/an

[30] Fast Access to Subscriber Terminals (FAST)—Physical Layer Specification, document Rec. ITU-T G.9701, Apr. 2017.

[31] W. Lanneer, P. Tsiaflakis, J. Maes, and M. Moonen, "Low-complexity nonlinear zero-forcing precoding under per-line power constraints for improved downstream G.fast active-user peak-rates," IEEE Trans. Commun., vol. 66, no. 6, pp. 2696-2707, Jun. 2018.

[32] Q. H. Spencer, A. L. Swindlehurst, and M. Haardt, "Zero-forcing methods for downlink spatial multiplexing in multiuser MIMO channels," IEEE Trans. Signal Process., vol. 52, no. 2, pp. 461-471, Feb. 2004.

[33] R. F. H. Fischer, Precoding and Signal Shaping for Digital Transmission. New York, NY, USA: JW, 2002.

[34] G. Ginis and J. M. Cioffi, "Vectored transmission for digital subscriber line systems," IEEE J. Sel. Areas Commun., vol. 20, no. 5, pp. 1085-1104, Jun. 2002.

[35] M. Joham and W. Utschick, "Ordered spatial Tomlinson-Harashima precoding," in Smart Antennas-State-of-the-Art (EURASIP Book Series on Signal Processing and Communications). New York, NY, USA: Hindawi Publishing Corporation, 2005.

[36] M. Hekrdla, A. Matera, W. Wang, D. Wei, and U. Spagnolini, "Ordered tomlinson-harashima precoding in G.fast downstream," in Proc. IEEE Global Commun. Conf. (GLOBECOM), Dec. 2015, pp. 1-6.

[37] R. E. Burkard, E. Çela, P. M. Pardalos, and L. S. Pitsoulis, "The quadratic assignment problem," in Handbook of Combinatorial Optimization. Boston, MA, USA: Springer, 1998, pp. 1713-1809.

[38] S. Sahni and T. Gonzalez, "P-complete approximation problems," J. ACM, vol. 23, no. 3, pp. 555-565, 1976.

[39] C. H. Papadimitriou and D. Wolfe, "The complexity of facets resolved," in Proc. 26th Annu. Symp. Found. Comput. Sci. (SFCS), Portland, OR, USA, 1985, pp. 74-78. 
[40] E. L. Lawler, "The quadratic assignment problem," Manage. Sci., vol. 9, no. 4, pp. 586-599, 1963.

[41] S. J. Russell and P. Norvig, Artificial Intelligence: A Modern Approach. London, U.K.: Pearson Education, 2016.

[42] G. R. Maccartney, T. S. Rappaport, S. Sun, and S. Deng, "Indoor office wideband millimeter-wave propagation measurements and channel models at 28 and $73 \mathrm{GHz}$ for ultra-dense 5G wireless networks," IEEE Access, vol. 3, pp. 2388-2424, 2015.

[43] B. Ai et al., "On indoor millimeter wave massive MIMO channels: Measurement and simulation," IEEE J. Sel. Areas Commun., vol. 35, no. 7, pp. 1678-1690, Jul. 2017.

[44] H. K. Bizaki and A. Falahati, "Tomlinson-Harashima precoding with imperfect channel state information," IET Commun., vol. 2, pp. 151-158, Jan. 2008.

[45] J. Maes, C. Nuzman, and P. Tsiaflakis, "Sensitivity of nonlinear precoding to imperfect channel state information in G.fast," in Proc. 24th Eur. Signal Process. Conf. (EUSIPCO), Aug. 2016, pp. 290-294.

[46] A. El Gamal and Y.-H. Kim, Network Information Theory. Cambridge, U.K.: Cambridge Univ. Press, 2011. 